OPEN ACCESS

Edited by:

Maria José Rodríguez Lagunas,

University of Barcelona, Spain

Reviewed by:

Ce Tang,

Tokyo University of Science, Japan

Enzo Spisni,

University of Bologna, Italy

*Correspondence:

Pengfei Chen

pfchen@sibs.ac.cn

Shaodong Luan szkidney3@163.com

Hanchao Gao

hcgao@foxmail.com

${ }^{\dagger}$ These authors have contributed equally to this work

Specialty section:

This article was submitted to

Mucosal Immunity,

a section of the journal

Frontiers in Immunology

Received: 31 July 2021 Accepted: 29 September 2021 Published: 14 October 2021

Citation:

Gao H, Cao M, Yao Y,

Hu W, Sun $H$, Zhang $Y$,

Zeng C, Tang J, Luan S and Chen P (2021) Dysregulated Microbiota-

Driven Gasdermin D Activation

Promotes Colitis Development

by Mediating IL-18 Release.

Front. Immunol. 12:750841. doi: 10.3389/fimmu.2021.750841

\section{Dysregulated Microbiota- Driven Gasdermin D Activation Promotes Colitis Development by Mediating IL-18 Release}

\author{
Hanchao $\mathrm{Gao}^{1 * t}$, Mengtao Cao ${ }^{1 \dagger}$, Yikun $\mathrm{Yao}^{2}$, Wenjun $\mathrm{Hu}^{3}$, Huimin Sun ${ }^{1}$, \\ Yingwei Zhang ${ }^{1}$, Changchun Zeng ${ }^{1}$, Jia Tang ${ }^{4}$, Shaodong Luan ${ }^{1 *}$ and Pengfei Chen ${ }^{1 *}$ \\ 1 Department of Medical Laboratory, Shenzhen Longhua District Central Hospital, Affiliated Central Hospital of Shenzhen \\ Longhua District, Guangdong Medical University, Shenzhen, China, ${ }^{2}$ Molecular Development of the Immune System Section, \\ Laboratory of Immune System Biology, and Clinical Genomics Program, National Institute of Allergy and Infectious Diseases \\ (NIAID), National Institutes of Health, Bethesda, MD, United States, ${ }^{3}$ Department of Anesthesiology, 305 Hospital of People's \\ Liberation Army of China (PLA), Beijing, China, ${ }^{4}$ National Health Commission (NHC), Key Laboratory of Male Reproduction and \\ Genetics, Guangdong Provincial Reproductive Science Institute (Guangdong Provincial Fertility Hospital), Guangzhou, China
}

The balance between gut microbiota and host is critical for maintaining host health. Although dysregulation of the gut microbiota triggers the development of various inflammatory diseases, including colitis, the molecular mechanism of microbiota-driven colitis development is largely unknown. Here, we found that gasdermin D (GSDMD) was activated during acute colitis. In the dextran sulfate sodium (DSS)-induced colitis model, compared to wild-type mice, Gsdmd-deficient mice had less colitis severity. Mechanistically, GSDMD expression in intestinal epithelial cells (IECs), but not infiltrating immune cells, was critical for GSDMD-mediated colitis progression. Moreover, commensal Escherichia coli (E. coll) largely overgrew during colitis, and then the dysregulated commensal E. coli mediated GSDMD activation. Furthermore, the activated GSDMD promoted the release of interleukin-18 (IL-18), but not the transcript or maturation level of IL-18, which in turn mediated goblet cell loss to induce colitis development. Thus, GSDMD promotes colitis development by mediating IL-18 release, and the microbiota can mediate colitis pathogenesis through regulation of GSDMD activation. Our results provide a potential molecular mechanism by which the microbiota-driven GSDMD activation contributes to colitis pathogenesis.

Keywords: gut microbiota, gasdermin D, colitis, intestinal epithelial cell, IL-18

\section{INTRODUCTION}

The gut microbiota constantly affects host nutrient absorption and immune system development (1, 2). The mutual relationship between the gut microbiota and host is crucial for maintaining intestinal homeostasis (3-5). When this homeostatic balance is compromised, excessive immune responses are triggered and consequently contribute to various inflammatory diseases, including 
inflammatory bowel disease (IBD) and colorectal cancer (4, 6-8). Various reports have shown that the dysregulation of gut microbiota and the host immune system is critical for IBD development $(3,4,9)$. However, it is still largely unknown how the dysregulated microbiota promotes IBD development.

IBD, including ulcerative colitis (UC) and Crohn's disease (CD), is a chronic inflammatory disease of the gastrointestinal tract characterized by abdominal pain, diarrhea, bloody stools and body weight loss $(8,10)$. Dextran sulfate sodium (DSS)induced colitis is a generally used mouse model to mimic human UC (11). The inflammasome is an intracellular multiprotein complex that induces mature interleukin-1 $\beta$ (IL-1 $\beta$ ) and IL-18 production and mediates cell pyroptosis (12). It has been reported that the inflammasome pathway is involved in IBD development (13). The major components and effector molecules of the inflammasome, such as NLRP3, ASC, AIM2, Caspase 1, Caspase 11 , IL-18, and IL-1 $\beta$, play important roles in DSSinduced colitis. However, controversial roles of some of these genes in colitis have been observed by different studies (14-27).

Recently, GSDMD was identified as another novel inflammasome effector that mediates cell pyroptosis by forming membrane pores $(28,29)$. When the canonical or noncanonical inflammasome is induced, GSDMD is activated and cleaved into the N-terminal domain (GSDMD-N) and Cterminal domain (GSDMD-C) by Caspase 1 and Caspase 11 in mice or Caspase 4/5 in humans. Inflammasome components, such as NLRP1, NLRP2, NLRP3, NLRP6, NLRC4 and NLRP9b, could activate Caspase 1, which in turn activated GSDMD to mediate canonical pyroptosis (30). In noncanonical infalmmasome, lipopolysaccharide (LPS) directly bound and activated Caspase 4/5/11, and then the activated Caspase 4/5/ 11 cleaved GSDMD to initiate pyroptosis. GSDMD-N, which is the active form of GSDMD, forms membrane pores to mediate mature IL- $1 \beta$ and IL-18 secretion as well as cell pyroptosis, while GSDMD-C has the opposite suppressive effect by binding GSDMD-N $(28,31,32)$.

Several studies have reported the role of GSDMD in neuron system. GSDMD mediated cell pyroptosis and promoted neuroinflammation in many nervous system diseases, including Parkinson's Disease (PD), Multiple Sclerosis (MS), spinal cord injury, stroke, Traumatic Brain injury (TBI) and Zika virusinduced brain atrophy $(30,33)$. Gsdmd deficient mice showed impaired neuroinflammation and pathogenesis of experimental autoimmune encephalomyelitis (EAE), a well-characterized animal model of MS (34). Liu et al, showed that ablation of Caspase 1 decreased TBI-induced pyroptosis (35). Baicalein, a flavonoid isolated from the traditional Chinese medicinal herbal Scutellaria baicalensis Georgi, reduced neuroinflammation in MPTP-induced PD mice through suppressing NLRP3/Caspase1/GSDMD Pathway (36). Moreover, other studies suggested that GSDMD might be a promising target for stroke therapy $(37,38)$. These researches indicate that GSDMD mediated-pyroptosis have played a critical role in neuroinflammation and nervous system diseases. Although GSDMD is a critical proinflammatory gene in neuron system, the in vivo role of GSDMD in intestinal inflammation remains unclear. Herein, our study found that dysregulated microbiota activated GSDMD, which in turn mediated DSS-induced colitis development by promoting IL-18 release.

\section{MATERIAL AND METHODS}

\section{Reagent and Cell Lines}

Anti-GSDMD and anti-IL-18 were purchased from Abcam (Cambridge, UK). Anti-GAPDH, anti-cleaved Caspase 3, and anti-Caspase 3 were purchased from Cell Signaling Technology (Danvers, MA, US). Anti-Caspase 11 was purchased from Novus (Littleton, CO, USA). Anti-Caspase 1(p20) was purchased from AdipoGen (San Diego, CA, USA). Anti-HA was from Biolegend (San Diego, CA, USA). HT-29 cells were maintained in DMEM containing $10 \%$ (vol/vol) FBS, penicillin $(100 \mathrm{U} / \mathrm{ml})$ and streptomycin $(100 \mu \mathrm{g} / \mathrm{ml})$.

\section{Mice}

Gsdmd $d^{-/-}$mice on the C57BL/6 background were purchased from GemPharmatech (Nanjing, China). All mice were maintained in specific pathogen-free conditions and littermates from the same mouse line were bred as strict controls. For the cohousing assay, 3-week old sex-matched wild-type (WT) and $G s d m d^{-/-}$mice were cohoused at a 1:1 ratio for 4 weeks before exposure to DSS and left together during colitis. All animal experiments were performed in compliance with the guide for the care and use of laboratory animals and were approved by the institutional biomedical research ethics committee of Guangdong Medical University.

\section{Induction of Colitis}

Six to eight-week-old WT and $G s d m d^{-/-}$mice (n=5 or 6/group) were given with 3\% DSS (Meilunbio, Dalian, China) in drinking water for 5 days, and then followed by normal drinking water until Day 8. Mice were sacrificed for tissue analyses on Day 8. For survival analysis, mice $(n=10)$ were given $3.5 \%$ DSS solution in drinking water for 5 days followed by normal drinking water until Day 15.

\section{Determination of Clinical Scores}

After the DSS challenge, fresh stool samples were collected on Day 6, and scoring for stool consistency and occult blood was performed as previously described $(7,8)$. In brief, stool scores were determined as follows: 0 , well-formed pellets; 1 , semiformed stools that did not adhere to the anus; 2 , semiformed stools that adhered to the anus; and 3, liquid stools that adhered to the anus. Bleeding scores were determined as follows: 0 , no blood by using hemoccult; 1 , positive hemoccult; 2 , blood traces in stool visible; 3 , gross rectal bleeding.

\section{Determination of Cell Proliferation and Death}

For determination of cell proliferation, paraffin sections were stained using anti-Ki67 antibody (Ebioscience, San Diego, CA, USA). For determination of cell death, a TUNEL assay was 
performed with the In situ Cell Death Kit (Roche, Mannheim, Germany) according to the manufacturer's recommendations. The positive cells were counted by light microscopy.

\section{Bone Marrow Chimeras}

Bone marrow transfer was used to create $G s d m d^{-/-}$chimera mice wherein the genetic deficiency of Gsdmd was confined to either circulating cells ( $G s d m d^{-1-} \rightarrow$ WT chimera) or nonhematopoietic tissue $\left(\mathrm{WT} \rightarrow G s d m d^{-/}\right)$. In brief, 6 to 8 week-old recipient WT control mice or $\mathrm{Gs}_{\mathrm{m}} \mathrm{m}^{-/-}$mice were lethally irradiated with 800 cGy, and then the recipient WT control mice or $\mathrm{Gsdm}^{-/-}$mice were injected in the tail vein with $5 \times 10^{6}-1 \times 10^{7}$ mixed bone marrow cells from donor WT mice or $G s d m d^{-/-}$mice. Four chimera groups were generated: $\mathrm{WT} \rightarrow \mathrm{WT} \quad(\mathrm{n}=6)$, $\mathrm{WT} \rightarrow G s d m d^{-/-}(\mathrm{n}=8), G s d m d^{-/-} \rightarrow \mathrm{WT} \quad(\mathrm{n}=6)$, and $G s d m d^{-/-}$ $\rightarrow$ Gsdmd $^{-1-}(\mathrm{n}=7)$. The transplanted mice were given drinking water containing $2 \mathrm{~g} / \mathrm{L}$ neomycin sulfate (Meilunbio) for 2 weeks. After 8 weeks of bone marrow reconstitution, colitis was induced in the mice with $2.5 \%$ DSS for 5 days, followed by normal drinking water until Day 8. Mice were sacrificed for tissue analyses on Day 8.

\section{Isolation of Intestinal Epithelial Cells and Lamina Propria Lymphocytes}

Isolation of intestinal epithelial cells (IECs) and lamina propria lymphocytes (LPLs) was previously described $(7,8)$. Briefly, the dissected colon tissues were washed in HBSS buffer and then cut into pieces and digested by $75 \mathrm{U} / \mathrm{ml}$ collagenase type XI (Sigma, St. Louis, MO, USA) and $20 \mu \mathrm{g} / \mathrm{ml}$ dispase (Sigma) in DMEM supplemented with $1 \%(\mathrm{vol} / \mathrm{vol}) \mathrm{FBS}$, penicillin $(100 \mathrm{U} / \mathrm{ml})$ and streptomycin $(100 \mu \mathrm{g} / \mathrm{ml})$ at $37^{\circ} \mathrm{C}$. After 3 hours of digestion, crypts containing IECs were isolated from the supernatant of the digestion buffer by centrifugation at $300 \mathrm{~g}$ for $5 \mathrm{~min}$. The isolated crypts were analyzed by immunoblot or RT-PCR.

For isolation of LPLs, the dissected colon tissues were washed in HBSS buffer, and the epithelium was removed by shaking at $250 \mathrm{rpm}$ shaking at $37^{\circ} \mathrm{C}$ in HBSS buffer containing $30 \mathrm{mM}$ EDTA, $1 \mathrm{mM}$ DTT and 5\% FBS for $30 \mathrm{~min}$. After sedimentation, the crypts containing supernatant were discarded and the remaining colon tissues were further cut into small pieces and digested by $200 \mathrm{U} / \mathrm{ml}$ collagenase type VIII (Sigma) and $150 \mu \mathrm{g} /$ $\mathrm{ml}$ DNase I (Sigma) in RPMI-1640 medium supplemented with $5 \%$ (vol/vol) FBS, penicillin $(100 \mathrm{U} / \mathrm{ml})$ and streptomycin (100 $\mu \mathrm{g} / \mathrm{ml})$ at $37^{\circ} \mathrm{C}$ for 1 hour. The supernatant was centrifuged at $450 \mathrm{~g}$ for $5 \mathrm{~min}$ to collect cells and the LPLs were further isolated by Percoll (40\%/80\%; Solarbio, Beijing, China). The isolated LPLs were then subjected to western-blotting or RTPCR analyses.

\section{Adenovirus-Mediated GSDMD-C Expression in Mice}

Mouse GSDMD-C with an HA tag was cloned into the pAdTrack-CMV vector and then recombined with the pAdEasy-1 vector. Recombinant Adv-GSDMD-C or empty vector (Adv-EV) was transfected into HEK 293A cells. Viruses were packaged and amplified as described (39). After titration,
$2 \times 10^{10}$ adenovirus particles were intraperitoneally injected into the indicated mice every other day. Four days later, colitis was induced in the mice with DSS solution as described.

\section{Recombinant Mouse IL-1 $\beta$ or IL-18}

Recombinant mouse IL-1 $\beta$ (Novoprotein, Shanghai, China) or PBS as negative control was injected into $\mathrm{Gsdmd}^{-/-}$mice intraperitoneally at a concentration of $0.5 \mu \mathrm{g}$ or $1 \mu \mathrm{g}$ per mouse in $200 \mu \mathrm{l}$ sterile PBS on Days 1, 2, 3, 4, 5, and 6 during DSS-induced colitis.

Recombinant mouse IL-18 (Novoprotein) or PBS as negative control was injected into $G s d m d^{-/-}$mice intraperitoneally at a concentration of $1 \mu \mathrm{g}$ per mouse in $200 \mu \mathrm{l}$ sterile PBS from Day 1 to Day 8 during DSS-induced colitis.

\section{Commensal Depletion}

The mice were treated with a cocktail of antibiotics in drinking water as previously reported (7). Briefly, mice were treated with of $1 \mathrm{mg} / \mathrm{ml}$ neomycin, $0.5 \mathrm{mg} / \mathrm{ml}$ vancomycin, $1 \mathrm{mg} / \mathrm{ml}$ metronidazole and $1 \mathrm{mg} / \mathrm{ml}$ ampicillin for 4 weeks. Every week fresh antibiotics were supplied. After 4 weeks, drinking water was further supplemented with $1 \mathrm{mg} / \mathrm{ml}$ streptomycin, $170 \mu \mathrm{g} / \mathrm{ml}$ gentamicin, $125 \mu \mathrm{g} / \mathrm{ml}$ ciprofloxacin, and $1 \mathrm{mg} / \mathrm{ml}$ bacitracin for one week. More than $99.9 \%$ of intestinal microbes were removed by this method. After mice were treated with antibiotics for 5 weeks, colitis was induced in the indicated mice as described in the section on the induction of colitis. For E. coli-induced GSDMD activation in vivo, antibiotic-treated mice were treated with 3\% DSS for 5 days, during which at Day 4 and Day 5 the mice were given $10^{10} \mathrm{CFU}$ of $E$. coli isolated from eosinmethylene blue (EMB) agar plates by gavage and rectal administration. The mice were sacrificed on Day 6, and colon tissues were obtained for further analysis.

\section{LPS Transfection and Cell Viability Measurement}

LPS was electroporated into HT-29 cells using the Neon Transfection System (Thermo Fisher Scientific, Waltham, MA, USA) following the manufacturer's instructions as previously reported $(28,40)$. Briefly, $1 \times 10^{6}$ HT-29 cells were transfected with $1 \mu \mathrm{g}$ LPS. Cell viability was measured at 2.5 hours after LPS transfection. Cell viability was determined by the CellTiter-Glo Luminescent Cell Viability Assay (Promega, Madison, WI, USA).

\section{RT-PCR}

Real-time PCR has been reported previously (41). Briefly, total RNA of cells or tissue was extracted with TRIzol ${ }^{\circledR}$ Reagent (Invitrogen, Shanghai, China). cDNA samples were synthesized with PrimeScript ${ }^{\text {TM }}$ RT Master Mix (Takara Bio, Dalian, China). The levels of genes of the interest were quantified using TB Green ${ }^{\circledR}$ Premix Ex Taq ${ }^{\mathrm{TM}}$ (Tli RNaseH Plus) (Takara Bio). The expression levels of the genes were calculated by the $2^{-\Delta \Delta C t}$ method and normalized to $\beta$-actin. Amplification of cDNA was performed using a ViiA 7 Real-Time PCR system (Applied Biosystems, Foster City, CA, USA) with the sequences of oligonucleotide primers shown in Supplementary Table 1. 


\section{Isolation of Bacterial Genomic DNA and Microbiota Analysis by Quantitative PCR}

Fresh fecal pellets were obtained at the indicated time points. Bacterial genomic DNA was extracted with the TIANamp Stool DNA Kit (Tiangen Biotech, Beijing, China) with the optional high-temperature step $\left(90^{\circ} \mathrm{C}\right)$ directly. To assess the abundance of specific intestinal bacterial groups, the extracted bacterial genomic DNA was analyzed with TB Green ${ }^{\circledR}$ Premix Ex Taq ${ }^{\text {TM }}$ (Tli RNaseH Plus) (Takara Bio) on a ViiA 7 Real-Time PCR System. Signals were normalized to universal bacteria, and normalized data were used to calculate relative levels of $16 \mathrm{~S}$ rRNA gene expression of indicated bacterial groups. The $16 \mathrm{~S}$ rDNA primer sequences are shown in Supplementary Table 2.

\section{Immunoblot Analysis}

The procedure of immunoblot analysis has been previously reported (42). In brief, the cells or tissues were lysed for $30 \mathrm{~min}$ in ice-cold RIPA lysis buffer supplemented with 10 $\mathrm{mM}$ sodium fluoride $(\mathrm{NaF}), 1 \mathrm{mM} \mathrm{Na} \mathrm{VO}_{4}, 1 \mathrm{mM}$ phenylmethylsulfonyl fluoride, and a complete protease inhibitor cocktail (Roche) and separated by SDS-PAGE. After the proteins were transferred onto polyvinylidene fluoride (PVDF) membranes (Millipore, Darmstadt, Germany), the PVDF membranes were blocked at room temperature for 1 hour and then incubated with primary antibody overnight at $4^{\circ} \mathrm{C}$. After incubation with secondary antibody for 1 hour at room temperature, the blots were visualized with enhanced chemiluminescence (ECL) detection reagents (Millipore).

\section{Histology and Immunostaining}

The colon tissue was fixed in $4 \%$ paraformaldehyde (PFA) for 48 hours and then embedded in paraffin wax. For assessment of injury, 5-mm sections were stained with hematoxylin-eosin $(\mathrm{H} \& \mathrm{E})$. Alcian blue $(\mathrm{AB}) /$ periodic acid-Schiff (PAS) staining was used to assess goblet cells in the colon. To assess macrophage infiltration, sections were stained with rat antimouse F4/80 antibody (Abcam) followed by goat anti-rat biotin conjugate. After incubation with $\mathrm{ABC}$ reagent, stained sections were photographed by light microscopy.

\section{Enzyme-Linked Immunosorbent Assay}

To measure IL-1 $\beta$ and IL-18 in colon tissues, a part of the colon was weighed and homogenized mechanically in PBS containing $1 \%$ NP-40 and a complete protease inhibitor cocktail (Roche). The protein level of IL-1 $\beta$ or IL-18 in colon homogenate was measured with ELISA kits (R\&D Systems, Minneapolis, $\mathrm{MN}$; Sino Biological, Beijing, China) according to the manufacturer's instructions.

The procedure of measuring the cytokines from cultured colon explants has been described previously (21). In brief, colon tissue explants were obtained and rinsed with PBS three times and cultured for 24 hours in DMEM containing 10\% FBS, L-glutamine, $1 \% \mathrm{P} / \mathrm{S}$ and neomycin at $37^{\circ} \mathrm{C}$. The protein level of IL- $1 \beta$ or IL-18 in the supernatant of cultured colon tissue was measured with ELISA kits (R\&D Systems; Sino Biological) according to the manufacturer's instructions.

\section{Statistical Analysis}

Prism software (GraphPad Software) was used to perform statistical analysis and graph development. A two-tailed Student's t test was used to compare differences between two groups. Survival curves are presented using the Kaplan-Meier method, and significance was calculated by the log-rank (MantelCox) test. $p$ values $<0.05$ were considered significant.

\section{RESULTS}

\section{GSDMD Is Activated in DSS-Induced Colitis}

Mouse GSDMD was ubiquitously expressed in the examined tissues and was highly expressed in the liver, colon and small intestine (Figure 1A). We isolated intestinal epithelial cells (IECs) and lamina propria lymphocytes (LPLs) from mouse colon tissues, and we found that both the mRNA and protein levels of GSDMD in IECs were much higher than those of GSDMD in LPLs (Figures 1B, C), suggesting that GSDMD is mainly expressed in IECs. Although the mRNA or protein level of full length GSDMD (GSDMD-FL) was not significantly regulated in the DSS-induced colitis model (Figures 1D, E), GSDMD was strongly cleaved to activated GSDMD-N form (p30 fragment) on Day 3 and the activation decreased on Day 6 during DSS-induced colitis (Figure 1E). In addition to GSDMD-N, another p47 fragment was also cleaved during DSS-induced colitis (Figure 1E). We also found that the expression of Caspase 1 or Caspase 11 was dramatically induced on Day 3 in the DSS-induced colitis model (Figure 1E and Supplementary Figure 1). These data suggest that GSDMD is activated in the experimental colitis model.

\section{GSDMD Aggravates the Pathology of DSS-Induced Colitis}

Next, we asked whether GSDMD plays an important role in colitis development. To this end, Gsdmd-deficient mice, as well as their control mice, were assessed with the DSS-induced colitis model. We found that, after challenging these mice with 3.5\% DSS for 5 days, $60 \%$ of the WT littermate mice died within 15 days, while all Gsdmd $\mathrm{d}^{-/-}$mice survived (Figure 1F). Treating the mice with 3\% DSS for 5 days led to significant body weight loss in WT control mice, while the body weight loss was alleviated in Gsdmd-deficient mice (Figure 1G). The bleeding score and stool score, which indicate colitis severity, were much lower in $\mathrm{Gsdmd}^{-/-}$mice (Figure 1H), and consistently, the colon lengths were longer in $G s d m d^{-/-}$mice (Figures 1I, J). The pathology of the injured colons from WT mice was more severe than that of $G s d m d^{-/-}$mice as indicated by hematoxylin-eosin staining showing the structure of the colon (Figure 1K). Moreover, macrophage infiltration was remarkably reduced in Gsdmd ${ }^{-/}$ mice compared to WT mice (Figure 1L). The mRNA levels of inflammatory cytokines, such as IL-1 $\beta$, TNF- $\alpha$, KC, IL-6, IL-17, and MCP-1, were largely upregulated in WT mice, while the expression of these genes was only minor induced in $G s d m d^{-1-}$ mice (Figure 1M). These data indicate that GSDMD is essential for DSS-induced colitis. 
A

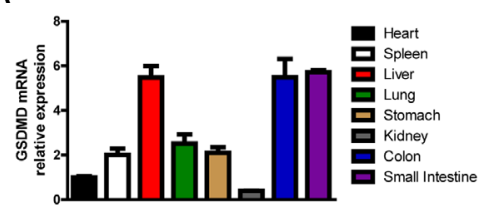

C

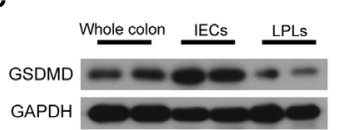

E

GSDMD-FL p $47 \rightarrow$

GSDMD-N

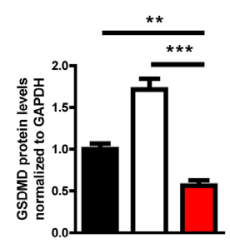

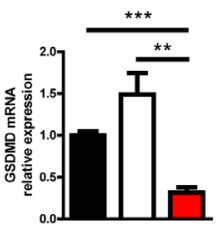

D
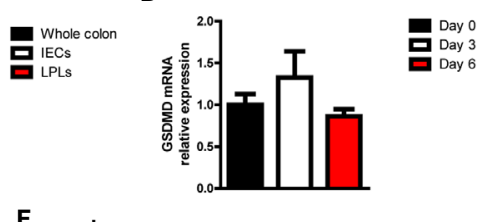

F

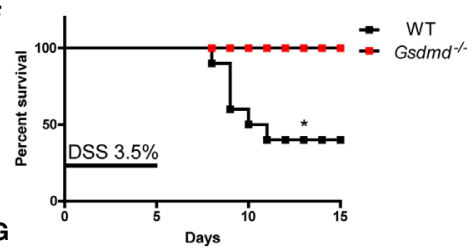

G

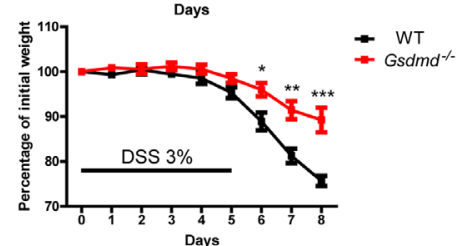

H

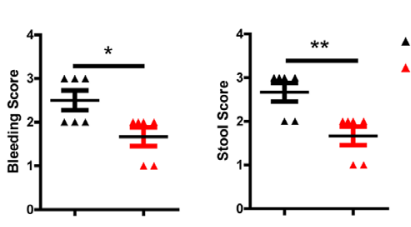

K

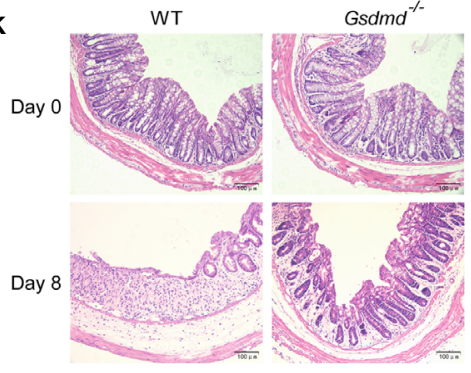

I

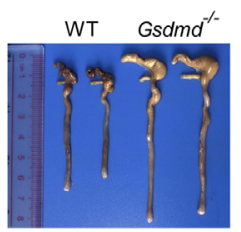

L

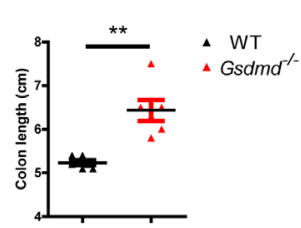

Gsdmd

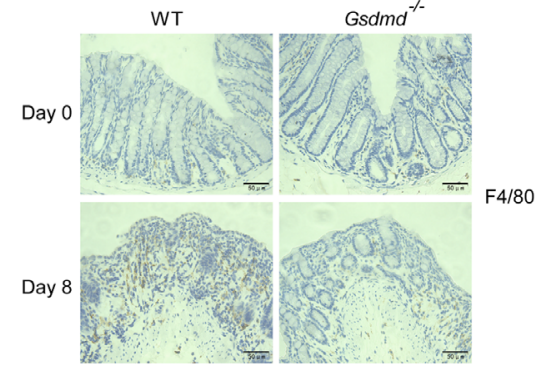

M
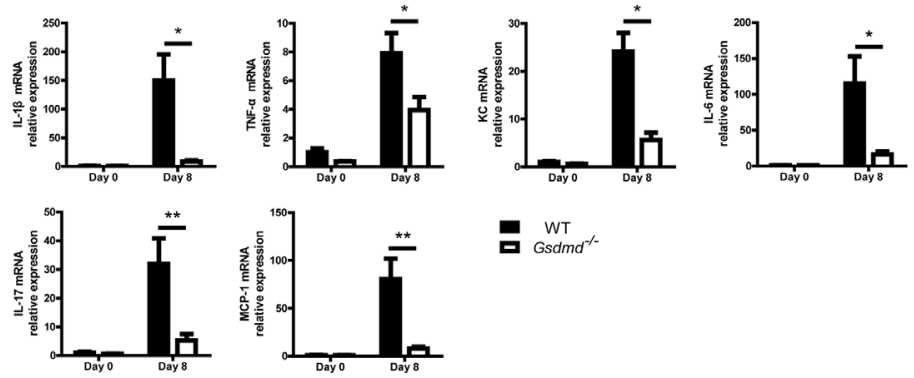

므 $\stackrel{W T}{\mathrm{Gs} T \mathrm{~m} d^{-1-}}$

FIGURE 1 | DSS-induced colitis is ameliorated in Gsdmd-deficient mice. (A) Quantitative mRNA expression of GSDMD in different C57BL/6 wild-type (WT) mouse tissues as indicated $(n=4)$. (B) Quantitative mRNA expression of GSDMD from WT mouse whole colon, IECs, or LPLs $(n=4)$. (C) Immunoblot analysis of GSDMD expression in WT mouse whole colon, IECs, or LPLs. The lower panel shows the quantitative analysis of GSDMD protein levels $(n=4)$. (D) Quantitative mRNA expression of GSDMD in WT mouse colons at the indicated times during DSS-induced colitis. (E) Immunoblot analysis of the expression of the indicated genes in the whole colon of WT mice at the indicated times during DSS-induced colitis. (F) Survival analysis of WT $(n=10)$ or Gsdmo ${ }^{-1}(n=10)$ mice treated with $3.5 \%$ DSS for 5 days. (G) Body weight change of WT $(n=6)$ or Gsdmd ${ }^{-1}(n=6)$ mice during the progression of DSS-induced colitis. (H) Bleeding score and stool score of the WT $(n=6)$ or Gsdmd $^{-1}(\mathrm{n}=6)$ mice on Day 6 of the colitis model as in (G). (I, J) Colon length (I) and the macroscopic view (J) of WT or Gsdmd ${ }^{-1-}$ mouse colons on Day 8 of the colitis model as in (G). (K) Hematoxylin and eosin (H\&E) staining of the representative colons from the mice on Day 0 or Day 8 of the colitis model as in (G) (200x magnification). (L) F4/80 staining of the representative colons from the WT or Gsdmd ${ }^{-1}$ mice on Day 0 or Day 8 of the colitis model as in (G) (400x magnification). (M) Quantitative mRNA expression of inflammatory genes as indicated from the colon of WT or Gsdmo ${ }^{-1-}$ mice on Day 0 or Day 8 of the colitis model as in (G). Data are representative of three (E-M) or four (A-D) independent experiments (mean \pm SEM in (A-D), (G-I) and (M). ${ }^{*} p<0.05,{ }^{* *} p<0.01,{ }^{* \star *} p<0.001$ by Student's t test. 
Moreover, to determine whether GSDMD affects IEC proliferation or IEC death during colitis, we analyzed intestinal cell proliferation and cell death by Ki67 and TUNEL staining, respectively. GSDMD deficiency reduced IEC proliferation and IEC death in DSS-induced colitis (Supplementary Figures 2A-D). GSDMD is the executor of cell pyroptosis, and TUNEL staining showed that cell death was reduced in $G s d m d^{-/-}$mice (Supplementary Figures 2C, D). To discriminate cell apoptosis and pyroptosis, we checked cleaved Caspase 3, a marker of cell apoptosis, and found that the level of cleaved Caspase 3 did not change between WT and Gsdmd $d^{-/-}$mice in DSS-induced colitis (Supplementary Figure 2E). These data show that GSDMD is most likely to mediate IEC pyroptosis during colitis.

Because antimicrobial peptides (AMPs) are critical in maintaining mucosal barrier integrity and suppressing colitis development, we investigated whether GSDMD promotes colitis by decreasing AMP production. We found that the mRNA levels of AMPs largely increased in WT mice treated with DSS. We found that the mRNA levels of AMPs in these mice were much higher than those in DSS-treated $G s d m d^{-/-}$mice (Supplementary Figure 3). The data suggest that AMPs are not the major factor causing GSDMD to promote colitis development.

\section{GSDMD Expression in Intestinal Epithelial Cells Is Critical for the Progression of Colitis}

To determine which cell populations are critical for GSDMDmediated DSS-induced colitis, we generated four groups of GSDMD bone marrow chimeras. Irradiated WT recipient mice receiving WT or $\mathrm{Gsdmd}^{-/-}$bone marrow had similar body weight loss (Figure 2A), clinical score (Figure 2B), colon length (Figures 2C, D), and histological damage (Figure 2E), suggesting that gut-infiltrating immune cells are not important for GSDMD-mediated colitis promotion. However, compared to the reconstituted WT recipient mice, irradiated $G s d m d^{-/-}$ recipient mice receiving WT or $G s d m d^{-1-}$ bone marrow showed reduced body weight loss (Figure 2A), clinical scores (Figure 2B), histological damage (Figure 2E), and increased colon length (Figures 2C, D), indicating that IECs are critical for the GSDMD-mediated promotion of colitis. Collectively, these data indicate that GSDMD expression in IECs is critical for GSDMD-mediated colitis promotion.

\section{GSDMD Activation Is Required for GSDMD-Mediated Colitis Development}

GSDMD-N can form membrane pores to mediate cell pyroptosis, while GSDMD-C can bind to GSDMD-N to maintain GSDMD in an unfunctional state $(28,31,32)$. Moreover, Shi et al, reported that overexpression of GSDMDC suppressed LPS-induced pyroptosis in HeLa cells due to transinhibition of endogenous GSDMD-N generated from caspase-4 cleavage (28). In our study, we found that adenovirus-mediated GSDMD-C (Adv-GSDMD-C) overexpression suppressed LPSinduced pyroptosis in HT-29 cells, an intestinal epithelial cell line (Supplementary Figures 4A, B). To determine whether the pathological role of GSDMD in acute colitis is dependent on
GSDMD activation, we used Adv-GSDMD-C overexpression in the mouse colon and then challenged the mice with DSS. The mice were intraperitoneally (i.p.) injected with empty control adenovirus (Adv-EV) or Adv-GSDMD-C every other day; 4 days later, the mice were challenged with DSS (Figure 3A). We found that GSDMD-C was significantly expressed in mouse colons that were infected by Adv-GSDMD-C several times (Supplementary Figure 4C and Supplementary Figure 5C). We detected the GSDMD expression and found that Adv-EV or Adv-GSDMD-C did not change GSDMD expression of mouse colon (Supplementary Figure 4D). The Adv-GSDMD-C treated mice had less body weight loss, and exhibited lower clinical scores, longer colon lengths and decreased colon injury than the Adv-EV treated mice (Figures 3B-E). The induced inflammatory genes also decreased in the Adv-GSDMD-C treated mice compared to the Adv-EV treated mice (Figure 3F). Next, we investigated whether Adv-GSDMD-C affects IEC pyroptosis during colitis. TUNEL staining showed that Adv-GSDMD-C largely suppressed IEC death (Supplementary Figures 5A, B). Immunoblot analysis showed that the expression of cleaved Caspase 3 was similar in both whole colon and IECs between Adv-EV- and Adv-GSDMD-C treated mice, suggesting that Adv-GSDMD-C suppressed IEC pyroptosis during DSSinduced colitis (Supplementary Figure 5C). Collectively, our data show that GSDMD-C protects mice from acute colitis.

\section{Gsdmd $^{-1-}$ Mice Have Fewer Intestinal Firmicutes, but This Is Not Associated With Their Hyposensitivity Toward DSS-Induced Colitis}

Various papers reported that gene-deficient mice had gut microbiota composition alterations, and some of the alterations were associated with IBD development (4, 20, 27, 43, 44). To determine whether Gsdmd ${ }^{-/}$mice have changed gut microbiota composition, we used $16 \mathrm{~S}$ rDNA real-time PCR to examine gut microbiota extracted from fecal samples of WT and $\mathrm{Gs}_{\mathrm{W}} \mathrm{md} \mathrm{d}^{-/-}$mice without DSS treatment. Four major intestinal bacterial phyla (Bacteroidetes, Firmicutes, Actinobacteria and Proteobacteria) and a colitis-associated bacteria phylum (TM7), as well as their representative classes, genera, or species, were checked $(7,44)$. Interestingly, the numbers of Actinobacteria, Bacteroidetes, Proteobacteria and TM7 were not significantly altered (Supplementary Figure 6A). A previous study reported that E. coli burden was obviously higher in inflammasome-deficient mice (27). However, we found that the E. coli burden was not significantly changed in $G s d m d^{-/-}$mice (Supplementary Figure 6A). Interestingly, the number of Firmicutes was reduced in Gsdmd-deficient mice (Supplementary Figure 6A). Segmented filamentous bacteria (SFB), Enterococcus and Lactobacillus, which belong to the Firmicutes phylum, were not altered in Gsdmddeficient mice; however, other members of Firmicutes, such as Clostridium cluster IV, Clostridium cluster XIVa, Eubacterium rectale (EREC), and Bacillus, which are potential beneficial microbiota for colitis (9), were obviously reduced in Gsdmd mice. To assess whether the altered composition of the gut microbiota is important for GSDMD-mediated colitis 
A
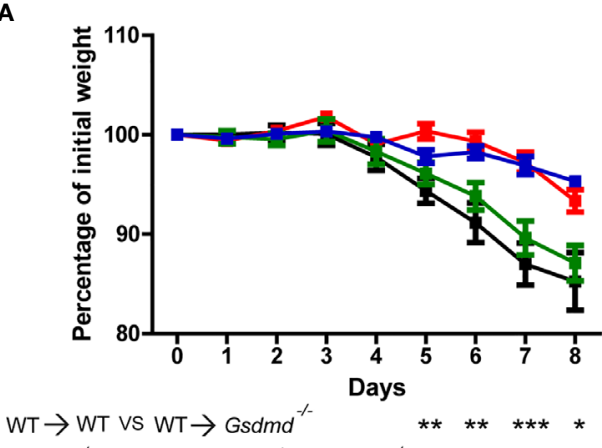
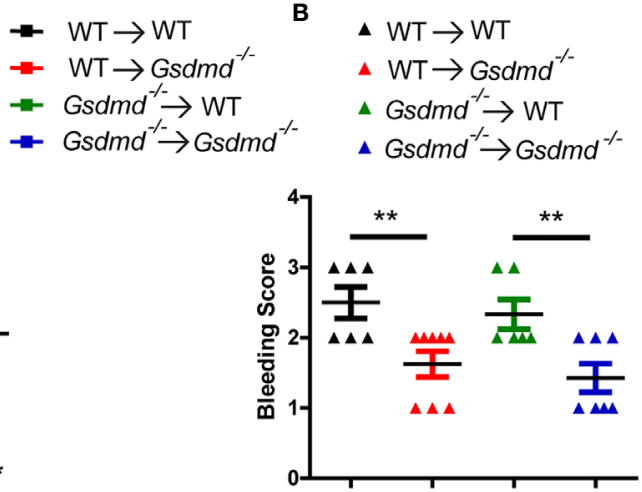

C

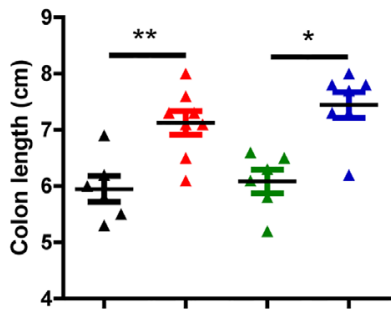

$\Delta \mathrm{WT} \rightarrow \mathrm{WT}$

$\Delta \mathrm{WT} \rightarrow \mathrm{Gsdmd}^{-/-}$

$\triangle \mathrm{Gsdmd} \stackrel{-1-}{\rightarrow} \mathrm{WT}$

A $\mathrm{Gsdmd}^{-/-} \rightarrow \mathrm{Gsdmd}^{-/-}$

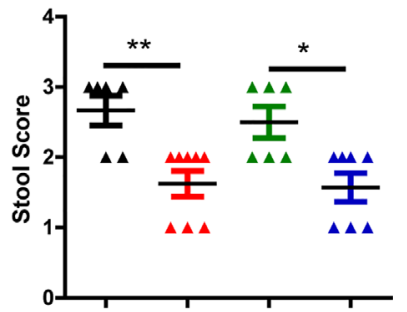

D

E
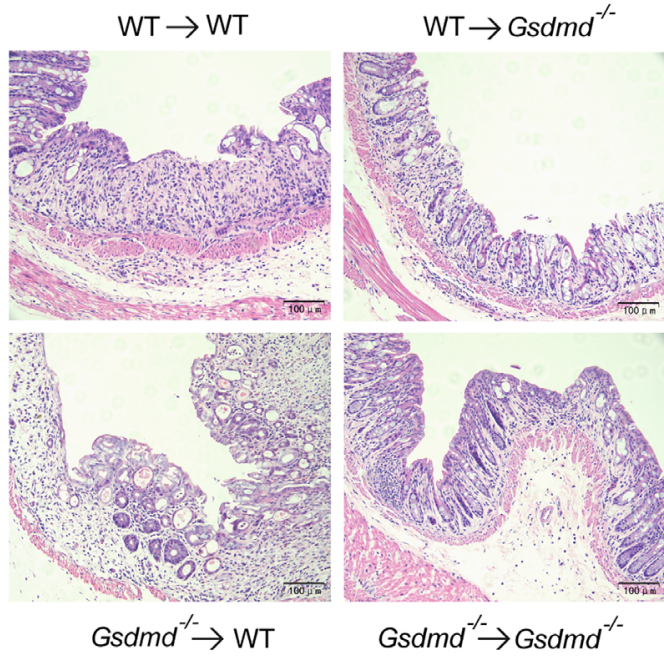

FIGURE 2 | GSDMD in nonhematopoietic cells is crucial for promoting DSS-induced injury. (A) During the progression of DSS-induced colitis, body weight

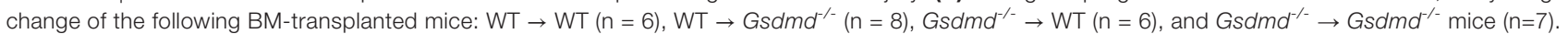
(B) Bleeding score and stool score of the transplanted mice on Day 6 of the colitis model as in (A). (C, D) Colon length (C) and the macroscopic view (D) of the transplanted mouse colon on Day 8 of the colitis model as in (A). (E) H\&E staining of the representative mouse colon on Day 8 of the colitis model as in (A) (200x magnification). Data are representative of two (A-E) independent experiments (mean \pm SEM in $\mathbf{A}-\mathbf{C}) .{ }^{*} p<0.05,{ }^{\star \star} p<0.01,{ }^{* \star *} p<0.001$ by Student's t test.

promotion, WT and Gsdmd ${ }^{-/-}$mice were cohoused for 4 weeks, and their gut microbiota were assessed before DSS challenge. As expected, the numbers of Firmicutes, Clostridium cluster IV, Clostridium cluster XIVa, EREC and Bacillus in Gsdmd-deficient mice equaled that of cohoused WT mice (Supplementary Figure 6B). In agreement with the results of separately housed mice (Figures 1G-K), despite similar gut microbiota compositions, cohoused Gsdmd ${ }^{-/}$mice showed reduced body weight loss compared to WT mice during DSS-induced colitis (Supplementary Figure 6C). The bleeding score, and stool score decreased and colon length increased in Gsdmd-deficient mice (Supplementary Figures 6D, E). Histological damage was also ameliorated in $\mathrm{Gsdmd}^{-/-}$mice (Supplementary Figure 6F). Together, these results indicate that although Gsdmd-deficient mice have fewer Firmicutes, this is not important for the reduced colitis severity in Gsdmd-deficient mice. 
A

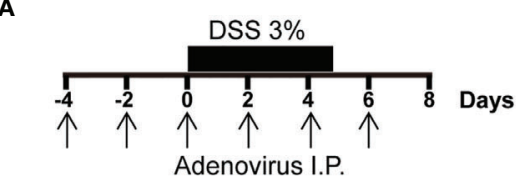

B

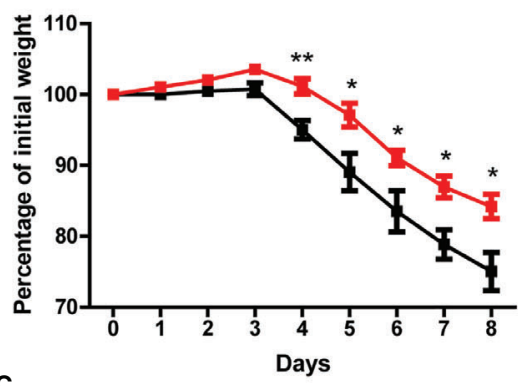

C
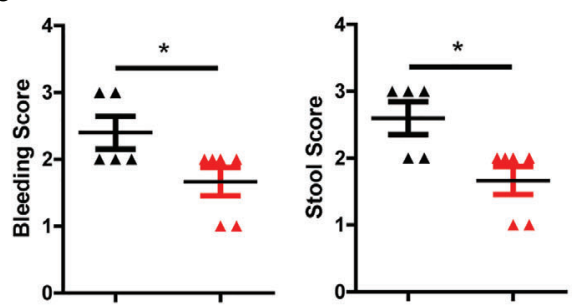
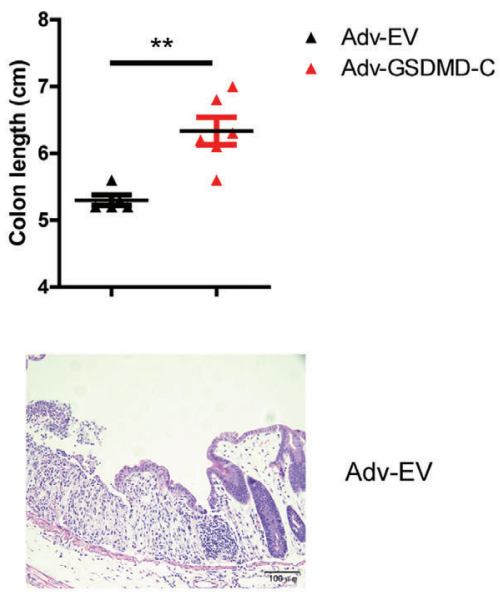

Adv-EV

Adv-EV

- Adv-GSDMD-C

$\mathbf{F}$
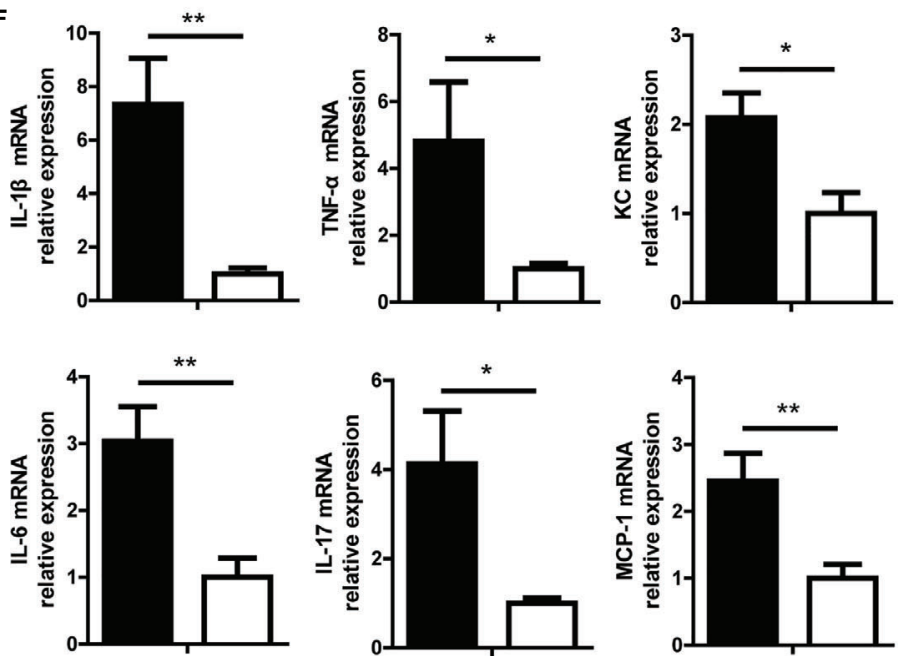

Adv-EV

Adv-GSDMD-C

Adv-GSDMD-C

FIGURE 3 | Adenovirus-mediated GSDMD-C expression protects mice from DSS-induced colitis. (A) Scheme of adenovirus-mediated GSDMD-C expression in mice. The mice were intraperitoneally (I.P.) injected empty control adenovirus (Adv-EV) or GSDMD-C expressing adenovirus (Adv-GSDMD-C) every other day, and 4 days later, colitis was induced in the mice with DSS solution. (B) Body weight change of WT mice treated with Adv-EV $(n=5)$ or Adv-GSDMD-C ( $n=6)$ during the progression of DSS-induced colitis. (C) Bleeding score and stool score of Adv-EV-or Adv-GSDMD-C-treated WT mice on Day 6 of the colitis model as in (B). (D, E) Colon length (D) and H\&E staining (E) of Adv-EV- or Adv-GSDMD-C-treated WT mouse colons on Day 8 of the colitis model as in (B) (200x magnification). (F) Quantitative mRNA expression of inflammatory genes as indicated from the colons of Adv-EV-or Adv-GSDMD-C-treated WT mice on Day 8 of the colitis model as in (B). Data are representative of three (B-F) independent experiments (mean \pm SEM in $\mathbf{B}-\mathbf{D}, \mathbf{F}$ ). ${ }^{*} p<0.05,{ }^{* *} p<0.01$ by Student's $t$ test.

\section{Dysregulated Commensal E. coli Activates GSDMD to Promote DSS-Induced Colitis}

Although the different microbiota compositions of WT and $\mathrm{Gs}_{\mathrm{N}} \mathrm{d}^{-/-}$mice are not important for GSDMD-mediated colitis development, the gut microbiota is dysregulated during acute colitis $(3,8)$. Therefore, we asked whether the activation of GSDMD is regulated by dysregulated gut microbiota during DSS-induced colitis. We removed gut microbiota with a cocktail of antibiotics (Abx), and we found that the expression of GSDMD-FL was not altered in the colons of WT mice treated 
with antibiotics during DSS-induced colitis; however, cleaved GSDMD-N was almost completely blocked (Figure 4A and Supplementary Figure 7A), which indicates that the gut microbiota contributes to GSDMD activation. We and others have previously reported that Enterobacteriaceae, primarily
Escherichia coli (E. coli), which belongs to Enterobacteriaceae, largely overgrew during acute colitis $(7,8,45,46)$, to determine which bacterial species are responsible for GSDMD activation, we asked whether the overgrowth E. coli is responsible for GSDMD activation. In agreement with previous reports, we found that the
A

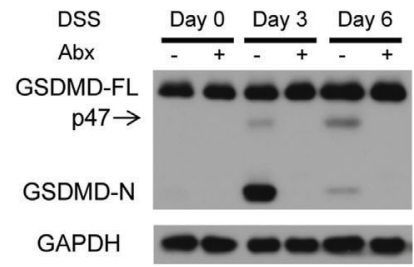

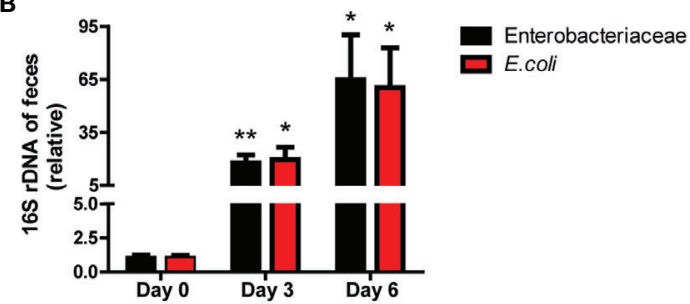

C
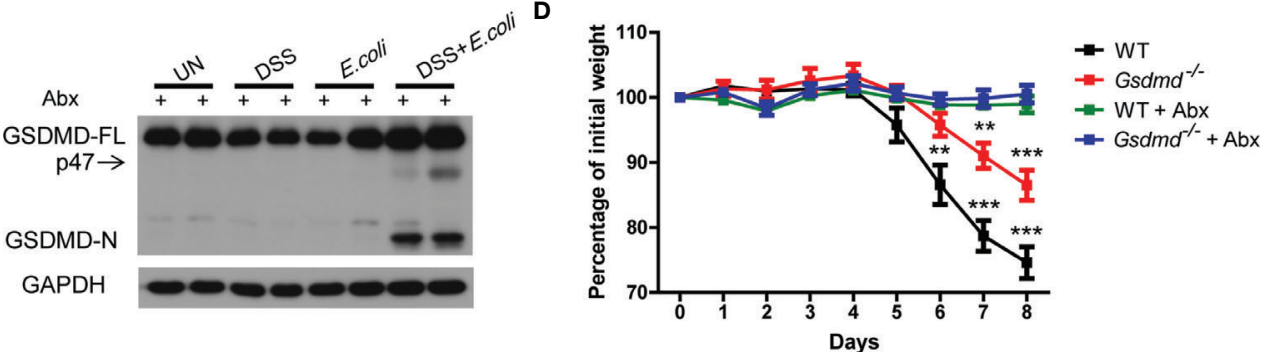

E

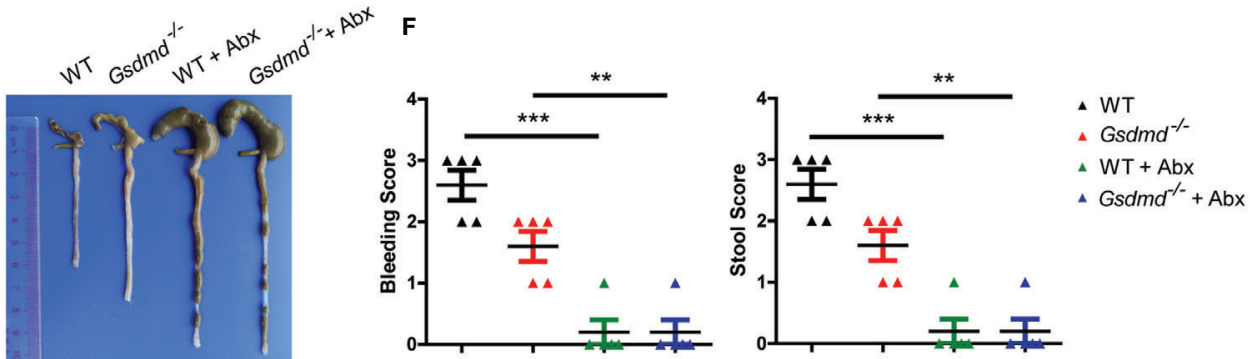

G

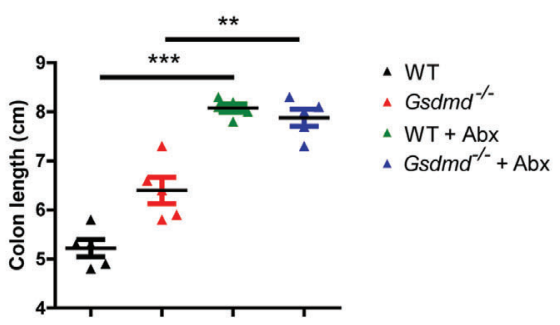

H

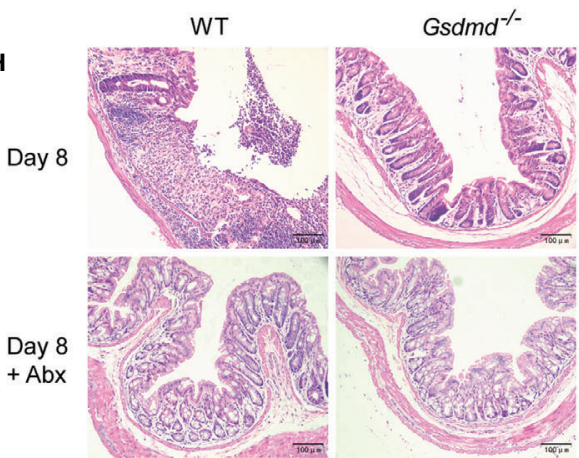

FIGURE 4 | In DSS-induced colitis, the dysregulated microbiota is responsible for GSDMD activation. (A) Immunoblot analysis of GSDMD expression in whole colons of WT mice treated with or without a cocktail of antibiotics (Abx) at the indicated times during DSS-induced colitis. (B) Real-time PCR analysis of 16S rRNA genes of Enterobacteriaceae or $E$. coli species in the feces of WT mice at the indicated times in the colitis model ( $n=6$ or $7 /$ group). (C) Immunoblot analysis of GSDMD expression from whole colons of Abx-treated WT mice, which were given with or without DSS solution or $10^{10} \mathrm{CFU}$ of $E$. coli, on Day 6 during DSS-induced colitis. (D) Body weight change of WT or Gsdmd ${ }^{-1-}$ mice treated with or without Abx during the progression of DSS-induced colitis ( $\left.n=5 / \mathrm{group}\right)$. (E) Macroscopic view of the representative mouse colon on Day 8 of the colitis model as in (D). (F) Bleeding score and stool score of WT or Gsdmo'-1- mice treated with or without Abx on Day 6 of the colitis model as in (D) ( $n=5 /$ group). (G) The colon length of WT or Gsdmd ${ }^{-1-}$ mice treated with or without Abx on Day 8 of the colitis model as in (D) ( $n=5$ /group). (H) H\&E staining of the representative mouse colon on Day 8 of the colitis model as in (D) (200x magnification). Data are representative of two (A-C) or three (D-H) independent experiments (mean \pm SEM in B, D, F, G). ${ }^{*} p<0.05,{ }^{* *} p<0.01,{ }^{* * *} p<0.001$ by Student's $t$ test. 
numbers of Enterobacteriaceae and E. coli largely increased in WT mice during colitis (Figure 4B). To determine whether the overgrowth of E. coli can activate GSDMD, we isolated E. coli from the feces of DSS-induced mice, and then the Abx-treated mice were given $E$. coli by gavage and rectal administrations. We found that DSS plus E. coli activated GSDMD in mice treated with antibiotics, whereas E. coli monocolonization alone did not (Figure 4C), which was consistent with our previous finding (7). These data suggest that the dysregulated microbiota, especially E. coli, is responsible for GSDMD activation during DSS-induced colitis.

Next, we investigated whether GSDMD is involved in microbiota-driven colitis development. We found that Abxtreated WT and $\mathrm{Gsdmd}^{-1}$ mice had almost no body weight loss during the DSS-induced colitis model (Figure 4D). Although untreated $\mathrm{Gsdmd}^{-/}$mice lost less body weight, and exhibited longer colons and lower clinical scores than untreated WT mice, microbiota depletion completely attenuated the phenotypic differences between WT and Gsdmd ${ }^{-/}$mice during DSS-induced colitis (Figures 4D-H). Consistently, microbiota removal also blocked the increased production of proinflammatory genes in both WT and Gsdmd $d^{--}$mice (Supplementary Figure 7B). These findings show that GSDMD is most likely critical for microbiotamediated contribution to colitis development. Collectively, our data suggest that dysregulated commensal E. coli mediates GSDMD activation, which in turn promotes colitis development.

\section{Reduced IL-1 $\beta$ Is Not Important for Protection Against Colitis in Gsdmd- Deficient Mice}

IL-1 $\beta$ is a critical proinflammatory cytokine produced by the inflammasome, and its role in colitis remains controversial (17, $24,25)$. We found that the production of IL-1 $\beta$ was reduced in $\mathrm{Gs}_{\mathrm{Cm}} \mathrm{d}^{-1-}$ mice compared to WT mice in DSS-induced colitis (Figure 1M and Figure 5A), so we asked whether the decreased IL- $1 \beta$ expression is important for the protective effect in Gsdmd-
A

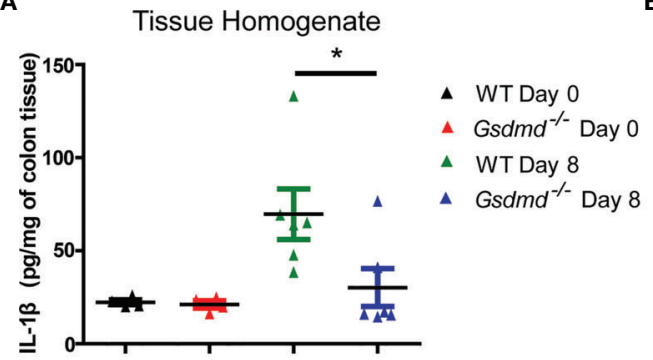

C

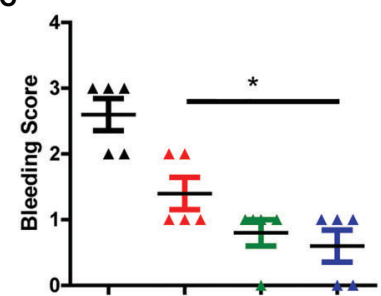

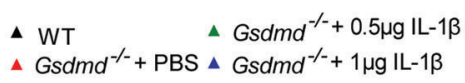

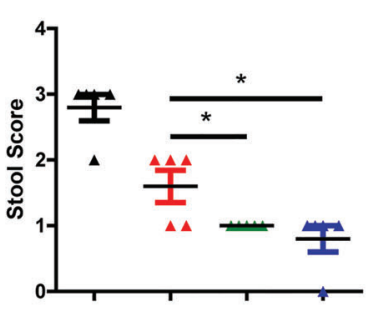

B

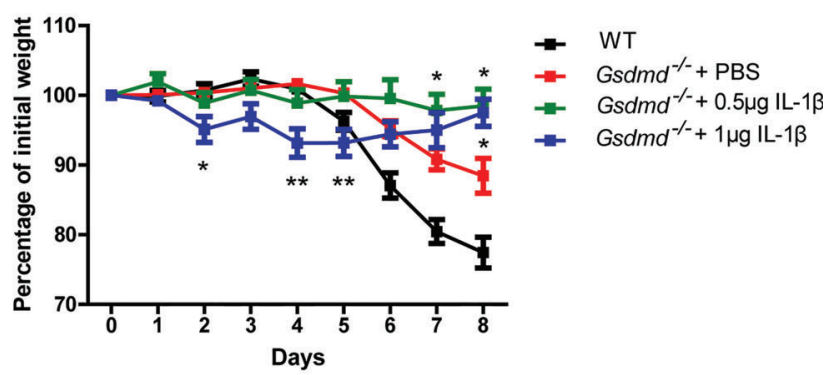

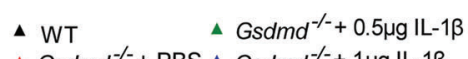

D

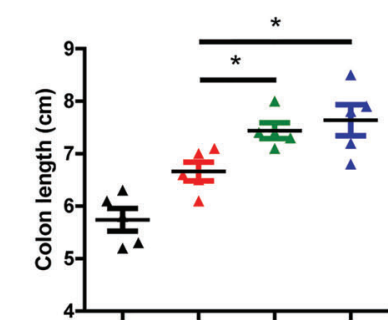

E

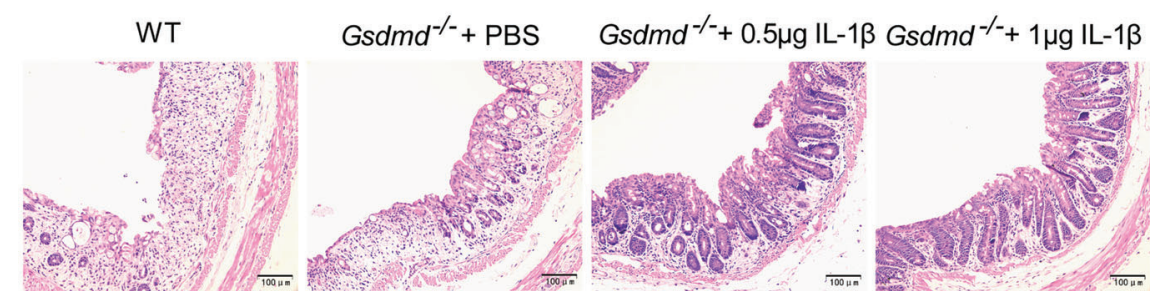

FIGURE 5 | Reduced IL-1 $\beta$ is not responsible for the protective effect against colitis in Gsdmd-deficient mice. (A) ELISA analysis of IL-1 $1 \beta$ protein levels in colon

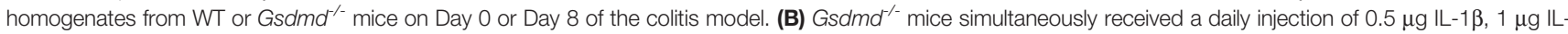
$1 \beta$ or PBS, while WT mice were injected with PBS ( $n=5 /$ group). The body weight change of the above mice was monitored during the progression of DSS-induced

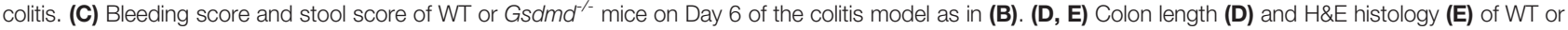
Gsdm ${ }^{-1 /-}$ mouse colons on Day 8 of the colitis model as in (B). Data are representative of two (A-E) independent experiments (mean \pm SEM in $\left.\mathbf{A}-\mathbf{D}\right)$. ${ }^{*} p<0.05$, ${ }^{* *} p<0.01$ by Student's t test. 
deficient mice. We intraperitoneally injected $G s d m d^{-/-}$mice with $0.5 \mu \mathrm{g}$ or $1 \mu \mathrm{g}$ recombinant mouse IL-1 $\beta$ every day (from Day 1 to Day 6) and challenged the mice with DSS. Surprisingly, we found that $1 \mu \mathrm{g}$ IL-1 $\beta$ treated Gsdmd $d^{-/-}$mice showed a slightly more body weight loss from Day 2 to Day 5 than PBS-treated $\mathrm{Gsdm}^{-/-}$mice; however, the $1 \mu \mathrm{g} \mathrm{IL}-1 \beta$-treated $\mathrm{Gs} d m \mathrm{~m}^{-/-}$mice exhibited obviously heavier body weight on Day 8 (Figure 5B). The $0.5 \mu \mathrm{g}$ IL-1 $\beta$-treated $G s d m d^{-1-}$ mice also showed heavier body weights on Day 8 and similar body weights on Day 2, Day 4 and Day 5 compared to PBS-treated Gsdmd $d^{-1-}$ mice (Figure 5B). Moreover, the IL-1 $\beta$ treated $G s d m d^{-/-}$mice showed lower clinical scores (Figure 5C), longer colon lengths (Figure 5D) and reduced colonic injury (Figure 5E). Collectively, our data indicate that exogenous IL-1 $\beta$ protects $G s d m d^{-1-}$ mice from acute colitis, and thus the decreased IL-1 $\beta$ is not the major reason for the protection against colitis in Gsdmd-deficient mice.

\section{GSDMD Promotes Acute Colitis Development by Mediating IL-18 Release}

IL-18 promotes DSS-induced colitis by driving goblet cell loss (19), and Miao et al, reported that GSDMD was responsible for IL-18 release in tubular epithelial cells during acute kidney injury (47). We asked whether GSDMD promotes IL-18 release during colitis. We found that the concentration of IL-18 in colon tissue homogenate or culture colon explant supernatant was significantly reduced in Gsdmd-deficient mice during colitis (Figure 6A). To determine whether the reduced IL-18 protein level is due to reduced IL-18 maturation or reduced IL-18 release, we checked intracellular mature IL-18 expression by immunoblots and found that the intracellular mature IL-18 level in Gsdmd-deficient mouse colons was greater than that in WT mouse colons, suggesting that the accumulation of intracellular mature IL-18 increases in Gsdmd-deficient colons (Figure 6B). Moreover, the mRNA level of IL-18 was not altered between WT and Gsdmd $d^{-/-}$mice (Figure 6C). These data suggest that the suppressed IL-18 release from the cytoplasm of Gsdm $\mathrm{d}^{-/-}$ mouse colon most likely accounts for the reduced IL-18 level in colon tissue homogenate or culture medium, and GSDMD might not affect the transcription or maturation of IL-18 during colitis. Next, we checked whether GSDMD affects goblet cell damage during DSS-induced colitis. As expected, compared to WT mice, the number of goblet cells in Gsdmd-deficient mice was better preserved during DSS-induced colitis (Figures 6D, E). During DSS-induced colitis, the mRNA level of Muc2, a mucin protein that was primarily produced by goblet cells, was higher in Gsdmd-deficient mice than in WT mice (Figure 6F). IL-18 drives goblet cell loss by preventing goblet cell maturation (19). In our setting, we indeed found that the mRNA levels of goblet cell maturation-related transcription factors Gfil, Spdef, and Klf4 were higher in Gsdmd-deficient mice than in WT mice during colitis (Figure 6F). To determine whether GSDMD activation is required for IL-18 release, we assessed IL-18 release in Adv-GSDMD-C treated mice. We found that the protein level of IL-18 from tissue homogenate or culture colon explant medium was decreased in GSDMD-C treated mice (Figure 6G). The intracellular mature IL-18 protein level was increased in the colon of Adv-GSDMD-C treated mice (Figure 6H). Meanwhile, the mRNA level of IL-18 was not altered (Figure 6I). These data suggest that GSDMD-C overexpression suppresses IL-18 release during DSS-induced colitis. Overexpression of GSDMD-C also increased the number of goblet cells in mice during colitis (Figures 6J, K) The mRNA levels of Gfil, Spdef and Klf4 also increased in AdvGSDMD-C treated mice (Figure 6L). Collectively, these data suggest that GSDMD promotes IL-18 release during colitis; and that the activation of GSDMD is required for its release.

To determine whether GSDMD exacerbates colitis pathology by inducing IL-18 release, we injected intraperitoneally Gsdmddeficient mice at a concentration of $1 \mu \mathrm{g}$ recombinant mouse IL18 per mouse daily during DSS colitis. We found that exogenous IL-18 obviously decreased $\mathrm{Gs} d \mathrm{md}^{-/-}$mouse body weight, and the reduced body weight was similar to that of WT mice (Figure 7A). The IL-18 treated Gsdmd $d^{-/-}$mice showed a higher clinical score (Figure 7B), shorter colon length (Figure 7C), and increased colonic injury (Figure 7D). Moreover, exogenous IL18 reduced the goblet cell number (Figures $7 \mathbf{E}, \mathbf{F})$, and decreased the mRNA levels of Gfil, Spdef, and Klf4 in Gsdmd deficient mice (Figure 7G). These data show that exogenous IL-18 promotes colitis severity in Gsdmd-deficient mice. Together, our data suggest that GSDMD promotes IL-18 release during colitis; and that the released IL-18 mediates colitis development by promoting goblet cell loss.

\section{DISCUSSION}

The interaction between the host and gut microbiota is critical for maintaining gut homeostasis $(4,48)$. When the homeostatic balance is compromised, the gut microbiota will be dysregulated and trigger the development of various inflammatory diseases, including colitis $(7,8,49)$. However, the molecular mechanism for microbiota-mediated regulation of colitis development is largely unknown. Here, we indicated that dysregulated microbiota-driven GSDMD activation promoted colitis development by inducing IL-18 release.

The roles of the inflammasome in colitis are controversial. Nlrp3- and Caspase 1-deficient mice were reported to show ameliorated colitis severity $(14,15,21)$, while others reported that DSS-induced colitis was aggravated in these mice $(16,22,23)$. Similarly, the roles of ASC, Caspase 11, IL-18, and IL-1 $\beta$ in colitis remain debatable $(17-21,24,25)$. GSDMD is another critical inflammasome protein that mediates cell pyroptosis $(28,29)$. Here, our data found that GSDMD deficiency protected mice from DSS-induced colitis, which is consistent with a very recent report (50). Interestingly, Ma et al. found that DSS-induced colitis was aggravated in Gsdmd-deficient mice (51). The possible reasons for the seemingly controversial data as follows: i) We used different strategies to induce colitis. Mice were treated with 3\% DSS for 5 days in our settings while 2.5\% DSS challenged for 6 days was used in their study. ii) The differences in mouse housing and feeding among animal facilities probably led to different gut microbiota compositions and then caused different colitis severities. 


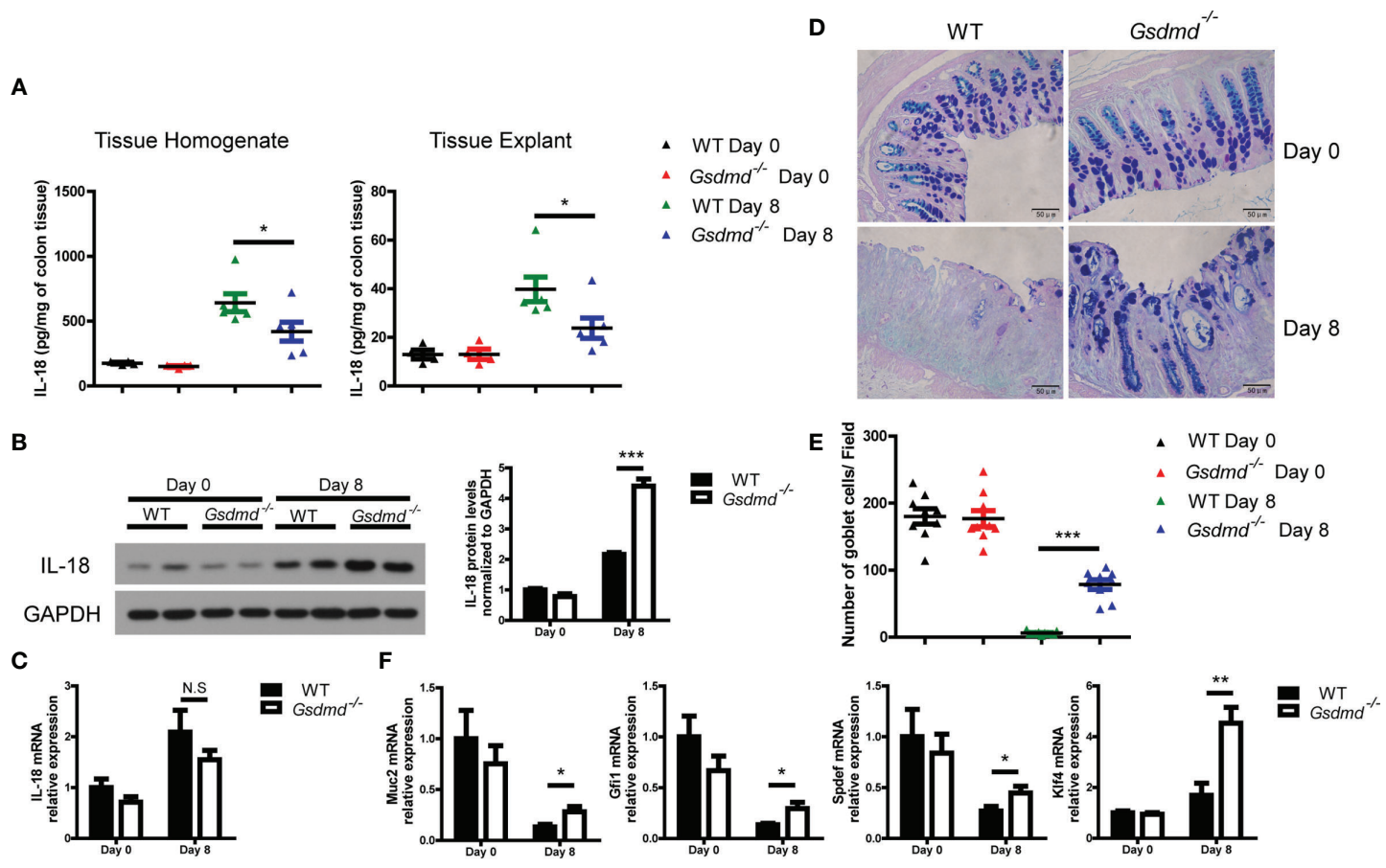

G
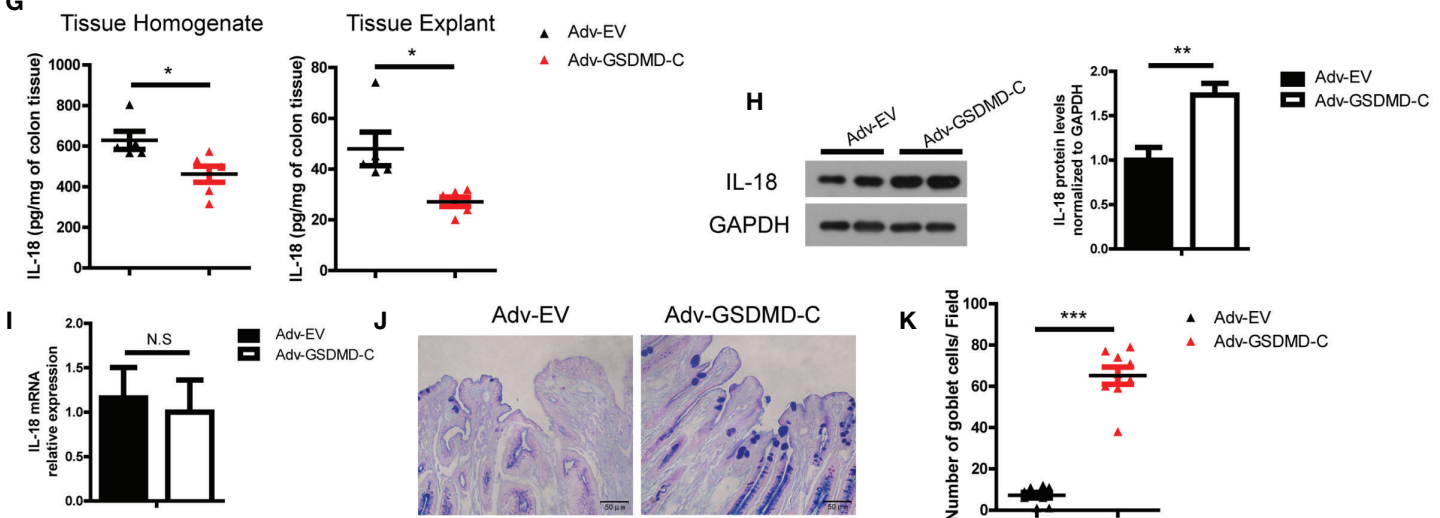

Adv-GSDMD-C
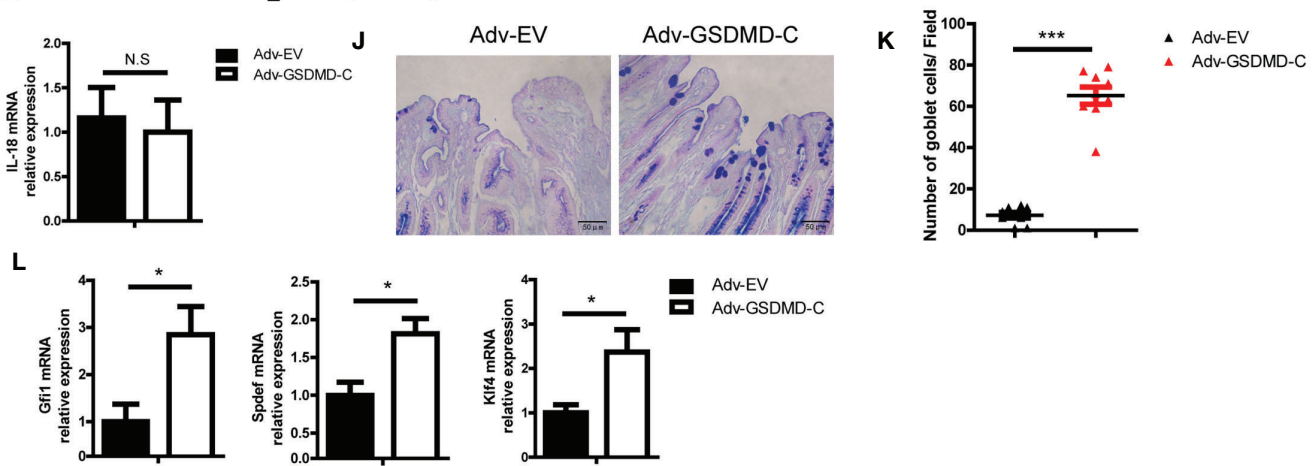

FIGURE 6 | GSDMD increases IL-18 release during DSS-induced colitis. (A) ELISA analysis of IL-18 protein levels in WT or Gsdmd ${ }^{-1-}$ mouse colon homogenates or cultured colon tissue on Day 0 or Day 8 of the colitis model, as shown in Figure 1G (n=4/6 per group). (B) Immunoblot analysis of IL-18 expression in WT or Gsdmd $^{-1-}$ mouse colons on Day 0 or Day 8 of the colitis model, as shown in Figure 1G. The quantitative analysis of IL-18 is shown on the right side. (C) Quantitative mRNA expression of IL-18 from WT or Gsdmd ${ }^{-1}$ mouse colons on Day 0 or Day 8 of the colitis model, as shown in Figure 1G. (D, E) AB-PAS staining of the representative colons from the mice on Day 0 or Day 8 of the colitis model, as shown in Figure $\mathbf{1 G}$ (400x magnification). The number of goblet cells per field (E) was determined as in (D) ( $n=9$ /group). (F) Quantitative mRNA expression of the indicated genes from the colon of WT or Gsdmd ${ }^{-1-}$ mice on Day 0 or Day 8 of the colitis model, as shown in Figure 1G. (G) ELISA analysis of IL-18 protein levels in the colons of Adv-EV- $(n=5)$ or Adv-GSDMD-C-treated $(n=6)$ WT mice on Day 8 of the colitis model, as shown in Figure 3B. (H) Immunoblot analysis of IL-18 expression in the colons of Adv-EV-or Adv-GSDMD-C-treated WT mice on Day 8 of the colitis model, as shown in Figure 3B. The quantitative analysis of IL-18 is shown on the right side. (I) Quantitative mRNA expression of IL-18 in the colons of Adv-EVor Adv-GSDMD-C-treated WT mice on Day 8 of the colitis model, as shown in Figure 3B. (J, K) AB-PAS staining of the representative colons from the Adv-EV- or Adv-GSDMD-C-treated WT mouse colon on Day 8 of the colitis model, as shown in Figure $\mathbf{3 B}$ (400x magnification). The number of goblet cells per field (K) was determined as in (J) ( $n=$ 9/group). (L) Quantitative mRNA expression of the indicated genes from the colons of Adv-EV- or Adv-GSDMD-C-treated WT mice on Day 8 of the colitis model, as shown in Figure 3B. Data are representative of two (A, B, G, H) or three (C-F, I-L) independent experiments (mean \pm SEM in $\mathbf{A}-\mathbf{C}, \mathbf{E}-\mathbf{I}$, $\mathbf{K}-\mathbf{L}) .{ }^{\star} p<0.05,{ }^{* \star} p<0.01,{ }^{* \star \star} p<0.001$ by Student's t test. N.S, no significance. 


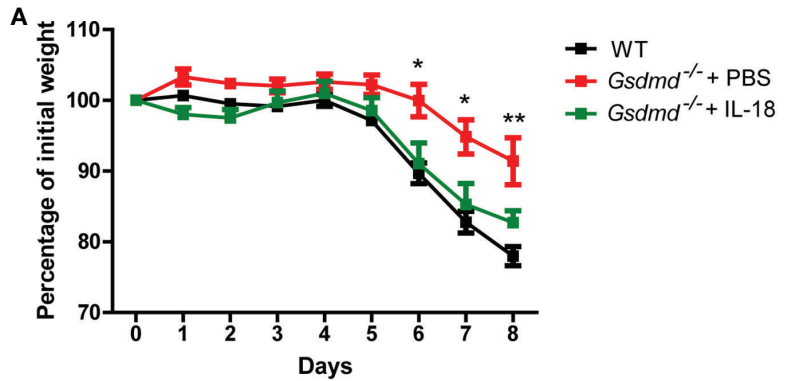

C

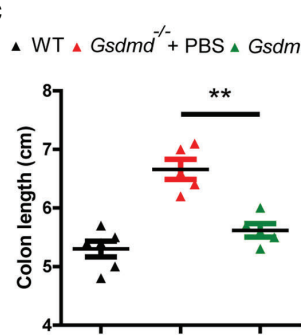

D

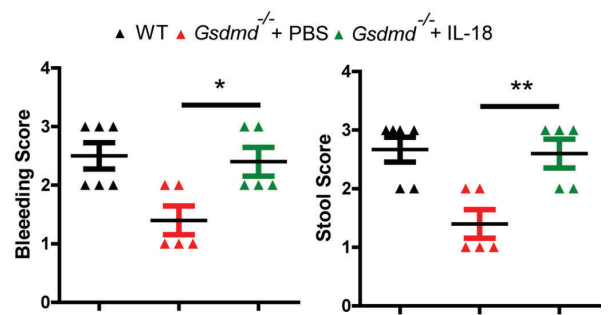

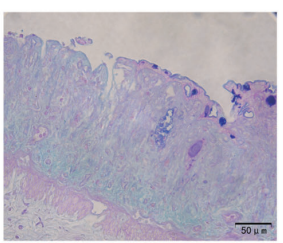

WT

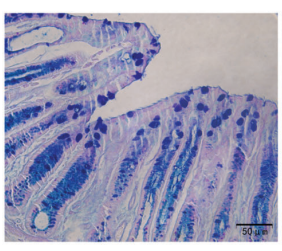

Gsdmd $^{-/-}+$PBS

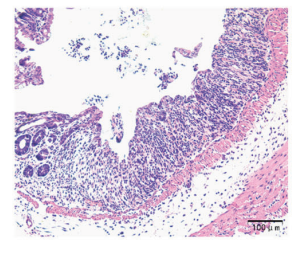

WT

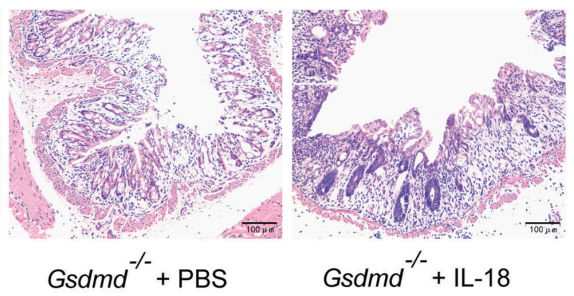

G
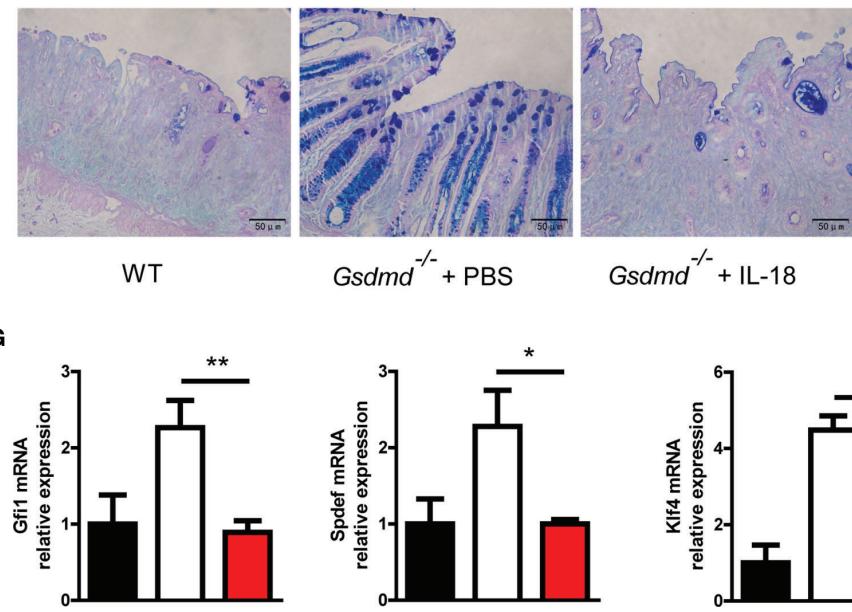

Gsdmd ${ }^{-/-}+\mathrm{IL}-18$

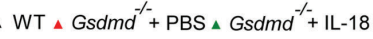

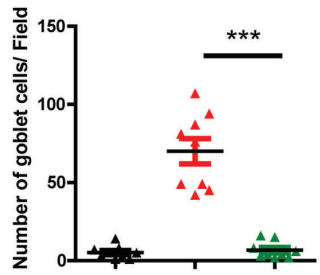

FIGURE 7 | IL-18 is involved in GSDMD-mediated colitis promotion. (A) Gsdmd ${ }^{-1}$ mice simultaneously received a daily injection of $1 \mu \mathrm{g}$ of IL-18 or PBS, while WT mice were injected with PBS ( $n=5 / 6$ per group). The body weight change of the above mice was monitored during the progression of DSS-induced colitis. (B) Bleeding score and stool score of the indicated mice on Day 6 of the colitis model as in (A). (C) Colon length of the indicated mice on Day 8 of the colitis model as in (A). (D) H\&E histology of representative mouse colons on Day 8 of the colitis model as in (A) (200x magnification). (E, F) AB-PAS staining of the representative colons from the mice on Day 8 of the colitis model as in (A) (400x magnification). The number of goblet cells per field (F) was determined as in (E) ( $n=9 / g r o u p)$. (G) Quantitative mRNA expression of the indicated genes from the mouse colon on Day 8 of the colitis model as in (A). Data are representative of two (A-G) independent experiments (mean \pm SEM in $\mathbf{A}-\mathbf{C}, \mathbf{F}-\mathbf{G}) .{ }^{\star} p<0.05,{ }^{\star *} p<0.01,{ }^{\star \star \star} p<0.001$ by Student's $t$ test.

IECs are the first lie of defense against pathogens and pathobionts, and IEC damage is the critical factor promoting colitis development (52). In the present study, we found that GSDMD was highly expressed in IECs but not LPLs. To investigate which cell populations are critical for GSDMDmediated colitis promotion, we used a bone-barrow transfer assay to generate GSDMD bone-barrow chimeras. Our data showed that GSDMD in IECs was responsible for GSDMDmediated colitis promotion, while gut infiltrating immune cellderived GSDMD was not involved in this process. Our findings further confirm that IECs play critical roles in inflammasomemediated colitis.

In our study, we found that GSDMD was activated in DSSinduced colitis. To investigate whether the activation of GSDMD is required for GSDMD-mediated colitis development, we used Adv-mediated GSDMD-C expression, which suppressed GSDMD-mediated cell pyroptosis by binding GSDMD-N, in WT mouse colons. We found that Adv-GSDMD-C obviously suppressed colitis development, suggesting that GSDMD activation is required for GSDMD-mediated colitis 
development. Furthermore, we investigated how GSDMD is activated in the mouse intestinal tract. Previously, we and others found that gram-negative bacteria Enterobacteriaceae, particularly E. coli species, largely overgrew during DSSinduced colitis and then exacerbated colitis pathology $(7,8,45$, 46). Here we found that Enterobacteriaceae and E. coli largely increased during DSS-induced colitis. When the gut microbiota, including E. coli, was removed by antibiotics, GSDMD activation was completely inhibited in the colon of DSS-treated mice. When the antibiotic-treated mice were transplanted with E. coli and challenged with DSS, the DSS plus E. coli group showed obvious GSDMD activation, while DSS or E. coli alone could not activate GSDMD. The data suggest that the E. coli translocates to the basolateral surface of IECs to directly induce GSDMD activation in IECs after DSS-mediated epithelium damage, while E. coli cannot activate GSDMD in IECs without DSS-induced epithelium damage (11). Collectively, these data suggested that the microbiota was dysregulated during colitis; and that the dysregulated microbiota, particularly E. coli, mediated colitis development by activating GSDMD.

IL- $1 \beta$ is an important proinflammatory cytokine produced by the inflammasome. Some reports found that IL-1 $\beta$ enhanced colitis severity in animal models $(24,25)$, while others reported that $I l 1 \beta^{-1-}$ and $I l 1 r I^{-/-}$, the IL- $1 \alpha$ and IL-1 $\beta$ receptor, mice displayed exacerbated severity in DSS-induced colitis $(17,53)$. We found that IL-1 $\beta$ production was obviously reduced in $G s d m d^{-/-}$mice during DSS-induced colitis, and we asked whether the reduced pathological severity of $\mathrm{Gsdmd}^{-/-}$mice was due to reduced IL-1 $\beta$ secretion. We injected $G s d m d^{-/-}$mice with recombinant IL-1 $\beta$ and challenged these mice with DSS and found that recombinant IL-1 $\beta$ did not increase the severity of colitis but ameliorated the disease in $G s d m d^{-/-}$mice. The possible reason is that IL-1 $\beta$ is involved in repairing IECs and reconstituting epithelial barriers during colitis, which is consistent with previous report (17). Interestingly, we found that $1 \mu \mathrm{g}$ IL-1 $\beta$-treated $\mathrm{Gs} d \mathrm{md}^{-/-}$mice showed more body weight loss on Day 2, Day 4 and Day 5 than the control group. The possible reason is that the IL- $1 \beta$-mediated intense inflammatory response leads to body weight loss, which is similar to TNF- or LPS-induced shock. Our data suggest that decreased IL-1 $\beta$ is not important for GSDMD-mediated colitis promotion.

The role of IL-18 in colitis is complicated. Some papers reported that IL-18 had a protective role in colitis $(16,23,54)$, and some other reports showed that IL-18 had a pro-colitogenic role in experimental colitis (55-57). Nowarski et al., reported that IL-18 suppressed goblet cell maturation and then promoted colitis development (19). In our study, we found that the protein level of IL-18 was reduced in the colon culture medium of $G s d m d^{-/-}$mice during DSS-induced colitis. Furthermore, we found that the reduced IL-18 in the colon culture medium was due to reduced IL-18 release but not reduced mRNA levels or decreased IL-18 maturation. Exogenous IL-18 increased colonic injury in $\mathrm{Gsdm}^{-/-}$mice during DSS-induced colitis. In addition, IL-18 reduced the goblet cell number in Gsdmd $d^{-/}$mice. These data indicate that GSDMD promotes colitis development by enhancing IL-18 release.
How does GSDMD promote IL-18 release? Miao et al., reported that Caspase 11 cleaved GSDMD into GSDMD-N during acute kidney injury, and then the cleaved GSDMD-N translocated onto plasma membrane to form membrane pores, which triggered cell proptosis and IL-18 release from primary cultured renal tubular epithelial cells (47). We hypothesized that GSDMD might play a similar role in acute kidney injury and acute colitis through a similar mechanism. Release of IL-18 was detected from both intestinal epithelial cells and lamina propria lymphocytes during colitis (19). Based on our data, we speculate that E. coli-derived LPS activated Caspase 11, which in turn cleaved GSDMD to GSDMD-N, forming membrane pores to release IL-18 from intestinal epithelial cells. More experiments will be performed to support the speculation in the near future.

In summary, we identified GSDMD as a critical procolitogenic gene. GSDMD is activated by the dysregulated microbiota and in turn mediates microbiota-driven colitis by promoting IL-18 release. Our study provides a novel mechanism for microbiota-mediated colitis development by activating GSDMD and suggests that GSDMD is a promising target for IBD therapy.

\section{DATA AVAILABILITY STATEMENT}

The original contributions presented in the study are included in the article/Supplementary Material. Further inquiries can be directed to the corresponding authors.

\section{ETHICS STATEMENT}

The animal study was reviewed and approved by Institutional Biomedical Research Ethics Committee of the Guangdong Medical University.

\section{AUTHOR CONTRIBUTIONS}

HG, MC, PC, and YY designed the experiments. HG and PC wrote the manuscript. $\mathrm{HG}, \mathrm{MC}$, and $\mathrm{PC}$ conducted the experiments and analyzed the data. $\mathrm{WH}$ and $\mathrm{HS}$ helped with experiments. YZ, CZ, and JT provided reagents and technical support. HG and SL supervised the study. All authors contributed to the article and approved the submitted version.

\section{FUNDING}

This work was supported by grants from the National Natural Science Foundation of China, Grant/Award Number: 81902021, 81902558, and 81801474, Guangdong Basic and Applied Basic Research Foundation, Grant/Award Number: 2019A1515011009, 2021A1515010683, 2020A1515010225, and 2021A1515010955, 
Shenzhen Foundation of Science and Technology, Grant/Award Number: JCYJ20180306172449376, JCYJ20180306172459580, and JCYJ20180306172502097, Shenzhen Longhua District Foundation of Science and Technology, Grant/Award Number: 201803, 2017006 and SZLHQJCYJ202002.

\section{REFERENCES}

1. Brestoff JR, Artis D. Commensal Bacteria at the Interface of Host Metabolism and the Immune System. Nat Immunol (2013) 14(7):676-84. doi: 10.1038/ni.2640

2. Kolodziejczyk AA, Zheng D, Elinav E. Diet-Microbiota Interactions and Personalized Nutrition. Nat Rev Microbiol (2019) 17(12):742-53. doi: 10.1038/s41579-019-0256-8

3. Caruso R, Lo BC, Nunez G. Host-Microbiota Interactions in Inflammatory Bowel Disease. Nat Rev Immunol (2020) 20(7):411-26. doi: 10.1038/s41577019-0268-7

4. Zhang M, Sun K, Wu Y, Yang Y, Tso P, Wu Z. Interactions Between Intestinal Microbiota and Host Immune Response in Inflammatory Bowel Disease. Front Immunol (2017) 8:942. doi: 10.3389/fimmu.2017.00942

5. Kostic AD, Howitt MR, Garrett WS. Exploring Host-Microbiota Interactions in Animal Models and Humans. Genes Dev (2013) 27(7):701-18. doi: $10.1101 / \operatorname{gad} .212522 .112$

6. Pickard JM, Zeng MY, Caruso R, Nunez G. Gut Microbiota: Role in Pathogen Colonization, Immune Responses, and Inflammatory Disease. Immunol Rev (2017) 279(1):70-89. doi: 10.1111/imr.12567

7. Song X, Gao H, Lin Y, Yao Y, Zhu S, Wang J, et al. Alterations in the Microbiota Drive Interleukin-17C Production From Intestinal Epithelial Cells to Promote Tumorigenesis. Immunity (2014) 40(1):140-52. doi: 10.1016/ j.immuni.2013.11.018

8. Song X, Dai D, He X, Zhu S, Yao Y, Gao H, et al. Growth Factor FGF2 Cooperates With Interleukin-17 to Repair Intestinal Epithelial Damage. Immunity (2015) 43(3):488-501. doi: 10.1016/j.immuni.2015.06.024

9. Zhang SL, Wang SN, Miao CY. Influence of Microbiota on Intestinal Immune System in Ulcerative Colitis and Its Intervention. Front Immunol (2017) 8:1674. doi: 10.3389/fimmu.2017.01674

10. Kaser A, Zeissig S, Blumberg RS. Inflammatory Bowel Disease. Annu Rev Immunol (2010) 28:573-621. doi: 10.1146/annurev-immunol-030409-101225

11. Chassaing B, Aitken JD, Malleshappa M, Vijay-Kumar M. Dextran Sulfate Sodium (DSS)-Induced Colitis in Mice. Curr Protoc Immunol (2014) 104:15 25 11-15 25 14. doi: 10.1002/0471142735.im1525s104

12. Opipari A, Franchi L. Role of Inflammasomes in Intestinal Inflammation and Crohn's Disease. Inflamm Bowel Dis (2015) 21(1):173-81. doi: 10.1097/ MIB.0000000000000230

13. Zhen Y, Zhang H. NLRP3 Inflammasome and Inflammatory Bowel Disease. Front Immunol (2019) 10:276. doi: 10.3389/fimmu.2019.00276

14. Siegmund B, Lehr HA, Fantuzzi G, Dinarello CA. IL-1 Beta -Converting Enzyme (Caspase-1) in Intestinal Inflammation. Proc Natl Acad Sci USA (2001) 98(23):13249-54. doi: 10.1073/pnas.231473998

15. Bauer C, Duewell P, Mayer C, Lehr HA, Fitzgerald KA, Dauer M, et al. Colitis Induced in Mice With Dextran Sulfate Sodium (DSS) Is Mediated by the NLRP3 Inflammasome. Gut (2010) 59(9):1192-9. doi: 10.1136/gut.2009.197822

16. Zaki MH, Boyd KL, Vogel P, Kastan MB, Lamkanfi M, Kanneganti TD. The NLRP3 Inflammasome Protects Against Loss of Epithelial Integrity and Mortality During Experimental Colitis. Immunity (2010) 32(3):379-91. doi: 10.1016/j.immuni.2010.03.003

17. Bersudsky M, Luski L, Fishman D, White RM, Ziv-Sokolovskaya N, Dotan S, et al. Non-Redundant Properties of IL-1alpha and IL-1beta During Acute Colon Inflammation in Mice. Gut (2014) 63(4):598-609. doi: 10.1136/gutjnl2012-303329

18. Demon D, Kuchmiy A, Fossoul A, Zhu Q, Kanneganti TD, Lamkanfi M. Caspase-11 Is Expressed in the Colonic Mucosa and Protects Against Dextran Sodium Sulfate-Induced Colitis. Mucosal Immunol (2014) 7(6):1480-91. doi: $10.1038 / \mathrm{mi} .2014 .36$

19. Nowarski R, Jackson R, Gagliani N, de Zoete MR, Palm NW, Bailis W, et al. Epithelial IL-18 Equilibrium Controls Barrier Function in Colitis. Cell (2015) 163(6):1444-56. doi: 10.1016/j.cell.2015.10.072

\section{SUPPLEMENTARY MATERIAL}

The Supplementary Material for this article can be found online at: https://www.frontiersin.org/articles/10.3389/fimmu.2021.750841/ full\#supplementary-material

20. Oficjalska K, Raverdeau M, Aviello G, Wade SC, Hickey A, Sheehan KM, et al. Protective Role for Caspase-11 During Acute Experimental Murine Colitis. J Immunol (2015) 194(3):1252-60. doi: 10.4049/jimmunol.1400501

21. Blazejewski AJ, Thiemann S, Schenk A, Pils MC, Galvez EJC, Roy U, et al. Microbiota Normalization Reveals That Canonical Caspase-1 Activation Exacerbates Chemically Induced Intestinal Inflammation. Cell Rep (2017) 19(11):2319-30. doi: 10.1016/j.celrep.2017.05.058

22. Allen IC, TeKippe EM, Woodford RM, Uronis JM, Holl EK, Rogers AB, et al. The NLRP3 Inflammasome Functions as a Negative Regulator of Tumorigenesis During Colitis-Associated Cancer. J Exp Med (2010) 207 (5):1045-56. doi: 10.1084/jem.20100050

23. Dupaul-Chicoine J, Yeretssian G, Doiron K, Bergstrom KS, McIntire CR, LeBlanc PM, et al. Control of Intestinal Homeostasis, Colitis, and ColitisAssociated Colorectal Cancer by the Inflammatory Caspases. Immunity (2010) 32(3):367-78. doi: 10.1016/j.immuni.2010.02.012

24. Cominelli F, Nast CC, Duchini A, Lee M. Recombinant Interleukin-1 Receptor Antagonist Blocks the Proinflammatory Activity of Endogenous Interleukin-1 in Rabbit Immune Colitis. Gastroenterology (1992) 103(1):6571. doi: 10.1016/0016-5085(92)91096-M

25. Seo SU, Kamada N, Munoz-Planillo R, Kim YG, Kim D, Koizumi Y, et al. Distinct Commensals Induce Interleukin-1beta via NLRP3 Inflammasome in Inflammatory Monocytes to Promote Intestinal Inflammation in Response to Injury. Immunity (2015) 42(4):744-55. doi: 10.1016/j.immuni.2015.03.004

26. Ratsimandresy RA, Indramohan M, Dorfleutner A, Stehlik C. The AIM2 Inflammasome Is a Central Regulator of Intestinal Homeostasis Through the IL-18/IL-22/STAT3 Pathway. Cell Mol Immunol (2017) 14(1):127-42. doi: $10.1038 / \mathrm{cmi} .2016 .35$

27. Hu S, Peng L, Kwak YT, Tekippe EM, Pasare C, Malter JS, et al. The DNA Sensor AIM2 Maintains Intestinal Homeostasis via Regulation of Epithelial Antimicrobial Host Defense. Cell Rep (2015) 13(9):1922-36. doi: 10.1016/ j.celrep.2015.10.040

28. Shi J, Zhao Y, Wang K, Shi X, Wang Y, Huang H, et al. Cleavage of GSDMD by Inflammatory Caspases Determines Pyroptotic Cell Death. Nature (2015) 526(7575):660-5. doi: 10.1038/nature15514

29. Liu X, Zhang Z, Ruan J, Pan Y, Magupalli VG, Wu H, et al. InflammasomeActivated Gasdermin D Causes Pyroptosis by Forming Membrane Pores. Nature (2016) 535(7610):153-8. doi: 10.1038/nature18629

30. McKenzie BA, Dixit VM, Power C. Fiery Cell Death: Pyroptosis in the Central Nervous System. Trends Neurosci (2020) 43(1):55-73. doi: 10.1016/ j.tins.2019.11.005

31. Kuang S, Zheng J, Yang H, Li S, Duan S, Shen Y, et al. Structure Insight of GSDMD Reveals the Basis of GSDMD Autoinhibition in Cell Pyroptosis. Proc Natl Acad Sci USA (2017) 114(40):10642-7. doi: 10.1073/pnas. 1708194114

32. Liu Z, Wang C, Rathkey JK, Yang J, Dubyak GR, Abbott DW, et al. Structures of the Gasdermin D C-Terminal Domains Reveal Mechanisms of Autoinhibition. Structure (2018) 26(5):778-784 e773. doi: 10.1016/ j.str.2018.03.002

33. Hu X, Chen H, Xu H, Wu Y, Wu C, Jia C, et al. Role of Pyroptosis in Traumatic Brain and Spinal Cord Injuries. Int J Biol Sci (2020) 16(12):204250. doi: 10.7150/ijbs.45467

34. Li S, Wu Y, Yang D, Wu C, Ma C, Liu X, et al. Gasdermin D in Peripheral Myeloid Cells Drives Neuroinflammation in Experimental Autoimmune Encephalomyelitis. J Exp Med (2019) 216(11):2562-81. doi: 10.1084/ jem.20190377

35. Liu W, Chen Y, Meng J, Wu M, Bi F, Chang C, et al. Ablation of Caspase-1 Protects Against TBI-Induced Pyroptosis In Vitro and In Vivo. J Neuroinflamm (2018) 15(1):48. doi: 10.1186/s12974-018-1083-y

36. Rui W, Li S, Xiao H, Xiao M, Shi J. Baicalein Attenuates Neuroinflammation by Inhibiting NLRP3/caspase-1/GSDMD Pathway in MPTP Induced Mice 
Model of Parkinson's Disease. Int J Neuropsychopharmacol (2020) 23 (11):762-73. doi: 10.1093/ijnp/pyaa060

37. Wang J, Yao J, Liu Y, Huang L. Targeting the Gasdermin D as a Strategy for Ischemic Stroke Therapy. Biochem Pharmacol (2021) 188:114585. doi: 10.1016/j.bcp.2021.114585

38. Chen S, Mei S, Luo Y, Wu H, Zhang J, Zhu J. Gasdermin Family: A Promising Therapeutic Target for Stroke. Trans stroke Res (2018) 9(6):555-63. doi: 10.1007/s12975-018-0666-3

39. Gao H, Cao M, Chen P, Cooper DKC, Zhao Y, Wei L, et al. TNF-Alpha Promotes Human Antibody-Mediated Complement-Dependent Cytotoxicity of Porcine Endothelial Cells Through Downregulating P38-Mediated Occludin Expression. Cell Communication Signaling CCS (2019) 17(1):75. doi: 10.1186/s12964-019-0386-7

40. Shi J, Zhao Y, Wang Y, Gao W, Ding J, Li P, et al. Inflammatory Caspases Are Innate Immune Receptors for Intracellular LPS. Nature (2014) 514 (7521):187-92. doi: 10.1038/nature13683

41. Gao H, Chen P, Wei L, Xu J, Liu L, Zhao Y, et al. Angiopoietin-1 and Angiopoietin-2 Protect Porcine Iliac Endothelial Cells From Human Antibody-Mediated Complement-Dependent Cytotoxicity Through Phosphatidylinositide 3-Kinase/AKT Pathway Activation. Xenotransplantation (2017) 24(4). doi: 10.1111/xen.12309

42. Cao M, Chen F, Xie N, Cao MY, Chen P, Lou Q, et al. C-Jun N-Terminal Kinases Differentially Regulate TNF- and TLRs-Mediated Necroptosis Through Their Kinase-Dependent and -Independent Activities. Cell Death Dis (2018) 9(12):1140. doi: 10.1038/s41419-018-1189-2

43. Li J, Butcher J, Mack D, Stintzi A. Functional Impacts of the Intestinal Microbiome in the Pathogenesis of Inflammatory Bowel Disease. Inflamm Bowel Dis (2015) 21(1):139-53. doi: 10.1097/MIB.0000000000000215

44. Elinav E, Strowig T, Kau AL, Henao-Mejia J, Thaiss CA, Booth CJ, et al. NLRP6 Inflammasome Regulates Colonic Microbial Ecology and Risk for Colitis. Cell (2011) 145(5):745-57. doi: 10.1016/j.cell.2011.04.022

45. Lupp C, Robertson ML, Wickham ME, Sekirov I, Champion OL, Gaynor EC, et al. Host-Mediated Inflammation Disrupts the Intestinal Microbiota and Promotes the Overgrowth of Enterobacteriaceae. Cell Host Microbe (2007) 2 (2):119-29. doi: 10.1016/j.chom.2007.06.010

46. Winter SE, Winter MG, Xavier MN, Thiennimitr P, Poon V, Keestra AM, et al. Host-Derived Nitrate Boosts Growth of E. Coli in the Inflamed Gut. Science (2013) 339(6120):708-11. doi: 10.1126/science.1232467

47. Miao N, Yin F, Xie H, Wang Y, Xu Y, Shen Y, et al. The Cleavage of Gasdermin D by Caspase-11 Promotes Tubular Epithelial Cell Pyroptosis and Urinary IL-18 Excretion in Acute Kidney Injury. Kidney Int (2019) 96 (5):1105-20. doi: 10.1016/j.kint.2019.04.035

48. Levy M, Thaiss CA, Elinav E. Metabolites: Messengers Between the Microbiota and the Immune System. Genes Dev (2016) 30(14):1589-97. doi: $10.1101 / \mathrm{gad} .284091 .116$

49. Brennan CA, Garrett WS. Gut Microbiota, Inflammation, and Colorectal Cancer. Annu Rev Microbiol (2016) 70:395-411. doi: 10.1146/annurev-micro102215-095513
50. Bulek K, Zhao J, Liao Y, Rana N, Corridoni D, Antanaviciute A, et al. EpithelialDerived Gasdermin D Mediates Nonlytic IL-1beta Release During Experimental Colitis. J Clin Invest (2020) 130(8):4218-34. doi: 10.1172/JCI138103

51. Ma C, Yang D, Wang B, Wu C, Wu Y, Li S, et al. Gasdermin D in Macrophages Restrains Colitis by Controlling cGAS-Mediated Inflammation. Sci $A d v$ (2020) 6(21):eaaz6717. doi: 10.1126/sciadv.aaz6717

52. Lei-Leston AC, Murphy AG, Maloy KJ. Epithelial Cell Inflammasomes in Intestinal Immunity and Inflammation. Front Immunol (2017) 8:1168. doi: 10.3389/fimmu.2017.01168

53. Gonzalez-Navajas JM, Law J, Nguyen KP, Bhargava M, Corr MP, Varki N, et al. Interleukin 1 Receptor Signaling Regulates DUBA Expression and Facilitates Toll-Like Receptor 9-Driven Antiinflammatory Cytokine Production. J Exp Med (2010) 207(13):2799-807. doi: 10.1084/jem.20101326

54. Salcedo R, Worschech A, Cardone M, Jones Y, Gyulai Z, Dai RM, et al. MyD88-Mediated Signaling Prevents Development of Adenocarcinomas of the Colon: Role of Interleukin 18. J Exp Med (2010) 207(8):1625-36. doi: 10.1084/jem.20100199

55. Siegmund B, Fantuzzi G, Rieder F, Gamboni-Robertson F, Lehr HA, Hartmann G, et al. Neutralization of Interleukin-18 Reduces Severity in Murine Colitis and Intestinal IFN-Gamma and TNF-Alpha Production. Am J Physiol Regulatory Integr Comp Physiol (2001) 281(4):R1264-1273. doi: 10.1152/ajpregu.2001.281.4.R1264

56. Ten Hove T, Corbaz A, Amitai H, Aloni S, Belzer I, Graber P, et al. Blockade of Endogenous IL-18 Ameliorates TNBS-Induced Colitis by Decreasing Local TNF-Alpha Production in Mice. Gastroenterology (2001) 121(6):1372-9. doi: 10.1053/gast.2001.29579

57. Kanai T, Watanabe M, Okazawa A, Sato T, Yamazaki M, Okamoto S, et al. Macrophage-Derived IL-18-Mediated Intestinal Inflammation in the Murine Model of Crohn's Disease. Gastroenterology (2001) 121(4):875-88. doi: 10.1053/gast.2001.28021

Conflict of Interest: The authors declare that the research was conducted in the absence of any commercial or financial relationships that could be construed as a potential conflict of interest.

Publisher's Note: All claims expressed in this article are solely those of the authors and do not necessarily represent those of their affiliated organizations, or those of the publisher, the editors and the reviewers. Any product that may be evaluated in this article, or claim that may be made by its manufacturer, is not guaranteed or endorsed by the publisher.

Copyright $\odot 2021 \mathrm{Gao}, \mathrm{Cao}, \mathrm{Yao}, \mathrm{Hu}$, Sun, Zhang, Zeng, Tang, Luan and Chen. This is an open-access article distributed under the terms of the Creative Commons Attribution License (CC BY). The use, distribution or reproduction in other forums is permitted, provided the original author(s) and the copyright owner(s) are credited and that the original publication in this journal is cited, in accordance with accepted academic practice. No use, distribution or reproduction is permitted which does not comply with these terms. 